\title{
Design of a Multi-epitope Vaccine Against Acinetobacter baumannii Using Immunoinformatics Approach
}

\author{
Maryam Touhidinia $^{1} \cdot$ Fatemeh Sefid $^{2,3} \cdot$ Mozhgan Bidakhavidi $^{1,4}$
}

Accepted: 17 July 2021 / Published online: 28 August 2021

(c) The Author(s), under exclusive licence to Springer Nature B.V. 2021

\begin{abstract}
Acinetobacter baumannii is one of the most successful pathogens causing nosocomial infections and has significantly multidrug-resistant. So far, there are no certain treatments to protect against infection with $A$. baumannii, therefore an effective A. baumannii vaccine needed. The purpose of this study was to predict antigenic epitopes of $C a r O$ protein for designing the A. baumannii vaccine using immunoinformatics analysis. $\mathrm{CarO}$ protein is one of the most important factors in the resistance against the antibiotic Carbapenem. In this study, T and B-cell epitopes of $\mathrm{CarO}$ protein were predicted and screened based on the antigenicity, toxicity, allergenicity features. The epitopes were linked by suitable linkers. Four different adjuvants were attached to the vaccine constructs which among them, vaccine construct 3 was chosen to predict the secondary and the $3 \mathrm{D}$ structure of the vaccine. The refinement process was performed to improve the quality of the 3D model structure; the validation process is performed using the Ramachandran plot and ProSA z-score. The designed vaccine's binding affinity to six various HLA molecules and TLR 2 and TLR4 were evaluated by molecular docking. Finally, in silico gene cloning was performed in the pET28a (+) vector. The findings suggest that the vaccine may be a promising vaccine to prevent $A$. baumannii infection.
\end{abstract}

Keywords Multi-epitope vaccine $\cdot$ Acinetobacter baumannii $\cdot \mathrm{CarO} \cdot$ Immunoinformatics

\section{Introduction}

Acinetobacter baumannii is an aerobic, pleomorphic, nonmotile, typically short, almost round, and rod-shaped Gramnegative bacterium (Perez et al. 2007). It can cause infections in parts of the body such as blood, urinary tract, and

Fatemeh Sefid

sefid.fateme@yahoo.com

Maryam Touhidinia

Maryamtohidi90@yahoo.com

Mozhgan Bidakhavidi

M_bidakhavidi@yahoo.com

1 Department of Biology, Faculty of Science, Yazd University, Yazd, Iran

2 Department of Medical Genetics, Shahid Sadoughi University of Medical Science, Yazd, Iran

3 Department of Biology, Science and Art University, Yazd, Iran

4 Department of Nursing, Nursing and Midwifery Research, Shahid Sadoughi University of Medical Sciences and Health Services, Yazd, Iran lungs (Eliopoulos et al. 2008). As an opportunistic pathogen, A. baumannii has a high incidence among patients in the intensive care unit (ICU) setting and immunocompromised individuals particularly those who have experienced a prolonged hospital stay (Valencia et al. 2009). In addition, the frequency of $A$. baumannii infections has been enhancing gently (Dijkshoorn et al. 2007). A. baumannii is able to grow at various temperatures and $\mathrm{pH}$ conditions. Also, this organism can exploits different types of both carbon and energy sources. These properties cause A. baumannii can persist in either moist or dry conditions in the hospital environment. This hardiness, combined with its resistance to many antimicrobial drugs, contributes to spread it in the hospital setting (Obeidat et al. 2014).

This phenomenon of multidrug-resistant (MDR) pathogens has become a serious concern about nosocomial and community infections (Dijkshoorn et al. 2007; Tohidinia et al. 2020). During 1970s, A. baumannii is thought to have been sensitive to wide range of antibiotics, but today the pathogen emerges immense resistance to most first-line antibiotics (Howard et al. 2012). Pathogenesis in A. baumannii infections is a result of several virulence factors such as 
porins, capsules, and cell wall lipopolysaccharide, enzymes, biofilm production, motility, and iron-acquisition systems (Eijkelkamp et al. 2014). These virulence factors contribute to the organism to resist in hard environmental conditions and enable development of intense infections (Ayoub Moubareck and Hammoudi Halat 2020). In addition, various resistance mechanisms in this pathogen are identified which resistance with major classes of antibiotics including penicillins, cephalosporins, carbapenems, most aminoglycosides, fluoroquinolones, chloramphenicol, and tetracyclines (Kyriakidis et al. 2021). These mechanism including wide array of antibiotic-hydrolyzing enzymes, efflux pump changes, impermeability, and antibiotic target mutations which gives a unique ability to the organism to maintain a multidrugresistant phenotype (Asif et al. 2018; Espinal et al. 2019). According to the report of the Centers for Disease Control and Prevention, in 2017, Carbapenem-resistant Acinetobacter caused an estimated 8500 infections in hospitalized patients, and 700 people died as a result. CDC has reported a list of urgent threats bacteria that have the danger of antibiotic resistance. This list includes Carbapenem-resistant Acinetobacter, Candida auris, Clostridioides difficile, Carbapenem-resistant Enterobacterales, Drug-resistant Neisseria gonorrhea. Carbapenem-resistant Acinetobacter has been reported in the first line of this serious danger (Prevention 2019).

Carbapenem is a category of highly effective antibiotic drugs generally used for the treatment of severe or high-risk bacterial infections (Shaker and Shaaban 2017). The growing trend of Carbapenem resistance in A. baumannii recently has become a grave concern around the world because it limits the range of therapeutic alternatives (Higgins et al. 2010). In the context of the increasing worldwide occurrence of Carbapenem-resistant $A$. baumannii strains, Non-enzymatic molecular mechanisms act in cooperation with Carbapenemhydrolyzing $\beta$-lactamases to provide an intense resistance. However, this process has not been described clearly (CatelFerreira et al. 2011). MDR strains showed a porin-mediated mechanism relating to the Carbapenem resistance-associated outer membrane protein, $\mathrm{CarO}$ (Catel-Ferreira et al. 2011; Mussi et al. 2007; Siroy et al. 2005). CarO protein generally is an eight-stranded $\beta$-barrel protein of $29 \mathrm{kDa}$ which plays a key role in selective uptake of the ornithine, other basic amino acids, and uptake of Carbapenem (Catel-Ferreira et al. 2011). Mutations in the $\mathrm{CarO}$ gene can alter the structure or decrease the expression of the porin, resulting in a decline of antibiotics entry into the bacteria (Poirel et al. 2006; Zahn et al. 2015). In This study CarO protein was evaluated by available bioinformatics tools for designing an efficient multi-epitope subunit vaccine for the induction of immune responses against $A$. baumannii infections.

One of the critical issues in immunization is the presence of epitopes including B cells, HTL, and CTL that are recognized by immune system cells and capable of stimulating an immune response (Catel-Ferreira et al. 2011). The epitopes are the parts of a protein that have two shapes, continuous and discontinuous epitopes. Discontinuous epitopes consist of the residues from distant parts of the sequence that collectively made up the epitope and they will usually not recognized individually by antibodies appointed against the discontinuous epitope, therefore these epitopes are not suitable for the multi-epitope vaccine. However, continuous epitopes, also called sequential epitopes, are conformation independent, so they have the ability to be recognized by antibodies individually and independently (Chandra and Singh 2012; Rubinstein et al. 2008). Epitopes can induce a more direct and potent immune response, than the response induced by the whole protein. Therefore, the identification of antigenic epitopes can be more effective and safe in vaccine design (Palatnik-de-Sousa et al. 2018).

Immunoinformatics plays a key role in vaccine design and antibody production (Kazi et al. 2018). In the past, antibody design and vaccine development are expensive and timeconsuming. Nowadays, advances in the field of bioinformatics have provided practical tools which can be used to lessen the time and cost for vaccine and antibody design (Anderson et al. 2013; Kazi et al. 2018). The Immunoinformatics approach allows the identification of the immunogenic epitopes from the pathogen genomes. Also, the ideal and immunogenic parts could be developed as potential vaccine candidates to trigger protective immune responses in the hosts (Ali et al. 2017).

Adjuvants are agents or drugs that have few or no antigenic effects or properties but can help enhance the efficacy and immunogenicity of vaccines (Mishra et al. 2020). In many studies, several kinds of adjuvants were used that had significant effects on the immunogenicity of the vaccine (Cayatte et al. 2017; Doener et al. 2019; Gasparini et al. 2001; Suschak et al. 2017; Yang et al. 2020). Adjuvants L7/ L12 ribosomal protein (Behbahani 2020; Saadi et al. 2017), beta-defensin (Gupta et al. 2020; Wang et al. 2020), HBHA protein (Kumar et al. 2019; Lei et al. 2020), and HBHA conserved sequence(Rashidian et al. 2020; Solanki and Tiwari 2018) recently been identified as efficient and helpful adjuvants, and we used them in this study.

Infections caused by A. baumannii are becoming extremely difficult to prevent and treat because infection control strategies against the spread of A. baumannii are facing new multidrug-resistant (Rasooli et al. 2020). This study identifies the potential and immunogenic epitopes of $\mathrm{CarO}$ protein by bioinformatics tools. Then, linkers and adjuvant were added to the selected epitopes to design final multiepitope vaccines and was selected the best vaccine construct based on allergenicity, antigenicity, and solubility features. The 3D structure of the protein was predicted, and its affinity to different HLA, TLR2, and TLR4 was investigated by 
molecular docking. Finally, the vaccine coding gene expression in E. coli was evaluated, and in silico cloning was performed in the pET28a (+) vector. This selected vaccine construct can be developed to cure the infection caused by the A. baumannii.

\section{Method}

\section{Sequence Availability and Alignment}

The $\mathrm{CarO}$ protein sequence was retrieved from NCBI at http://www.ncbi.nlm.nih.gov/protein and saved in FASTA format for further analyses (Pruitt et al. 2005); then Protein BLAST (http://blast.ncbi.nlm.nih.gov/Blast.cgi) against reference sequence on the database was performed using Blosum80 matrix to collect homologous sequences between different bacteria (Tatusova and Madden 1999). Because BLOSUM80 created for highly similar alignments and is used to find related proteins (Wheeler 2003). PRALINE server at https://www.ibi.vu.nl/programs/pralinewww/ was used for generating of alignments. This server based on homology-extended multiple alignments and the integration of predicted secondary structure information with iteration capabilities methods which have many advantages and have made this server stronger (Simossis and Heringa 2005). Alignments can demonstrate the conservancy of the protein residues among various strains (Simossis and Heringa 2003). The suitable vaccine candidate should able effective against all strains of a given pathogen. The conservancy of the amino acids among bacteria other than Acinetobacter implies probable cross-reactivity levels.

\section{Prediction T Cell MHC Class I Epitope}

The recognition of MHC-I antigenic peptide which exposed at the target cell surface is crucial for the adaptive immune response (Nguyen van Binh et al. 2006). NetCTL server at http://www.cbs.dtu.dk/services/NetCTL/ was used to predict MHC class I epitopes. In this server, the method integrates prediction of peptide MHC class I binding, proteasomal C terminal cleavage, and TAP transport efficiency (Larsen et al. 2005). Prediction threshold value 0.75 was set for epitope identification. Epitopes were chosen with a combinatorial score $>1$ for further analyses (Larsen et al. 2007). Also, IEDB server at http://tools.iedb.org/mhci/ was used to predict peptide binding to MHC class I molecules based on the consensus method. Identified T-cell epitopes with HLA alleles were selected by percentile rank and IC50 values. The lower the percentile rank was shown the higher interaction between the peptide and MHC molecules. In Addition, high binding epitopes have an IC50 value below $50 \mathrm{nM}$, and weak binding peptides and IC50 value below $500 \mathrm{nM}$. So, Epitopes with both IC50 value $<50 \mathrm{nM}$ and percentile rank $\leq 0.2$ were selected for more analyses (Vita et al. 2019).

\section{Prediction Class I Antigenic, Toxicity and Immunogenicity}

A second screening step including their important features such as antigenicity and allergenicity which were checked by AllergenFP server at http://ddg-pharmfac.net/AllergenFP/ and Vaxijen server at http://www.ddg-pharmfac.net/vaxijen/ VaxiJen/VaxiJen.html. AllergenFP is generally utilized for the prediction of allergenicity of epitopes for vaccine design (Dimitrov et al. 2014). VaxiJen server used to predict antigenic properties of the peptide at a threshold value of 0.5 . This server depends on Auto Cross Covariance (ACC) transformation, and alignment-independent predicted antigenic epitopes by their physicochemical behavior (Doytchinova and Flower 2007). In the following, Epitope/MHC complex should have the ability to evoke an immune response. So, we have used MHC I immunogenicity prediction tool in the IEDB server (Calis et al. 2013). Default parameters were selected and the epitopes which gained the positive value were chosen.

\section{Prediction T Cell MHC Class II Epitope and Analysis Antigenic and Toxicity}

To identify MHC-II binding epitopes two servers were employed. IEDB server at http://tools.iedb.org/mhci/ were used to compute MHC-II binding epitopes based on the consensus method. The consensus method uses a combination of both stabilization matrix alignment method and average relative binding matrix method. Predicted epitopes were selected relying on percentile rank and IC50 values (percentile rank $\leq 0.2$ and IC50 value $<50 \mathrm{nM}$ ) (Jensen et al. 2018). NetMHCII 2.3 server at http://www.cbs.dtu.dk/servi ces/NetMHCII/ was used to predict binding of peptides to HLA-DR, HLA-DQ and HLA-DP using artificial neural networks (Kaushik and Therapeutics 2019). Among predicted strong binding epitopes were chosen those had score $<1$ for further analyses. Antigenicity, toxicity, and allergenicity were evaluated for predicted epitopes. The antigenicity and allergenicity of the epitopes were calculated by VaxiJen and AllergenFP server respectively (Dimitrov et al. 2014; Doytchinova and Flower 2007). In addition, mutation and toxicity features were checked by ToxinPred server at http:// crdd.osdd.net/raghava/toxinpred/. In the silico method, ToxinPred was used to predict the toxicity of peptides and about how to design a peptide or protein with desired toxicity by mutating a minimum number of amino acids (Gupta et al. 2013). 


\section{Prediction and Screening of Liner B-Cell Epitopes}

The recognition of B-cell epitopes has a key role in vaccine design, immunodiagnostic tests, and antibody production. There are various servers to predict B cell epitopes. Since each server performs based on specific algorithms and exclusive methods in epitope prediction. Therefore, the usage of several tools to predict linear B-cell epitopes in protein sequences are more valid. Ellipro server at http:// tools.immuneepitope.org was employed to predict linear antibody epitopes based on a protein antigen's 3D structure (Ponomarenko et al. 2008). Also, liner B cell epitopes were predicted using an artificial neural network by ABCpred server at http://crdd.osdd.net (Saha and Raghava 2006a, b) In addition, SVMTriP (http://sysbio.unl.edu/SVMTriP/) was used in the prediction of the B cell linear epitopes. This server is a Support Vector Machine which used to predict linear antigenic epitopes which combine the Tri-peptide similarity and Propensity scores (SVMTriP) (Yao et al. 2012). For more identification of continuous B-cell epitopes from the $\mathrm{CarO}$ protein, Bcpred server at http://ailab.ist.psu.edu/ bcpred/predict.html was utilized and the epitopes size of 20 amino acids as well as the specificity threshold of $0.75 \%$ were considered. The Bcpred server for predicting employs a subsequence kernel-based SVM classifier (EL-Manzalawy et al. 2008). Selected epitopes from the servers mentioned above were screened for antigenicity and allergenicity properties (Dimitrov et al. 2014; Doytchinova and Flower 2007).

\section{Construction of Model Vaccine}

To construct the novel vaccine with low toxicity, allergenicity, and high immunogenicity, we have analyzed the different combinations of sequence constructs. During this novel multi-epitope subunit vaccine construction. Firstly, CTL, HTL, and B cell epitopes were joined with the help of amino acid linkers HEYGAEALERAG and GGGS respectively (Hasan et al. 2019; Nezafat et al. 2014). Secondly, for enhancing the immunogenicity of this sequence constructs were added the four different adjuvants L7/L12 ribosomal protein (Mallick et al. 2007), beta-defensin (Dar et al. 2015), HBHA protein (Nezafat et al. 2014), and HBHA conserved sequence respectively (Rana and Akhter 2016). Adjuvants sequences were added with the help of EAAAK linkers at both N- and C-terminal of Adjuvants (Saadi et al. 2017). PADRE peptide sequences were also incorporated along with the adjuvants by GGGS linker. PADRE peptide induced CD4+T-cells that improve efficacy and potency of peptide vaccine (Wu et al. 2010).

\section{Allergenicity, Antigenicity and Solubility Evaluation}

The Allergenicity of vaccine constructs was predicted by AlgPred server (https://webs.iiitd.edu.in/raghava/algpred/ submission.html) which based the SVM module on amino acid composition. A threshold score of prediction has been considered to be -0.40 . Prediction score less than the threshold value point displays the non-allergic feature of the peptide (Saha and Raghava 2006a, b). VaxiJen server and ANTIGENpro program (Kalita et al. 2020) at http://scratch. proteomics.ics.uci.edu were employed for the prediction of vaccine antigenicity behavior. In addition, SOLpro online server at http://scratch.proteomics.ics.uci.edu was utilized to predict the propensity of the protein to be soluble upon overexpression in Escherichia coli. This server uses a two-stage SVM architecture method to evaluate the protein solubility (Magnan et al. 2009).

\section{Secondary Structure Prediction}

The PSIPRED at http://bioinf.cs.ucl.ac.uk/psipred/ was used to predict the secondary structure of the vaccine construct. PSIPRED is a simple and accurate secondary structure prediction method, incorporating two feed-forward neural networks that perform an analysis on output obtained from PSIBLAST (McGuffin et al. 2000). In addition, Phyre2 server at http://www.sbg.bio.ic.ac.uk/ phyre2/html/page.cgi?id= index employed to validate the PSIPRED predictions (Kelley et al. 2015).

\section{Vaccine 3D Modeling, Refinement, and Validation}

Homology modeling is the construction of an atomic model of a purpose protein based solely on the target's amino acid sequence and the experimentally determined structures of homologous proteins, referred to as templates (Kelley et al. 2015). While $\mathrm{Ab}$ initio prediction is the challenging attempt to predict protein structures based only on sequence information and without using templates (Yang and Zhang 2015). There are many tools and servers that are used for homology modeling and $\mathrm{Ab}$ initio prediction. There is no single modeling program or server which is superior in every aspect to others (Dolan et al. 2011). The SWISS-MODEL server at http://swiss-model.expasy.org/ is a fully automated protein structure homology-modeling server (Kiefer et al. 2009). I-TASSER server at https://zhanglab.dcmb.med.umich. edu/I-TASSER/ is based on hierarchical method to protein structure prediction and structure-based function annotation (Zhang 2008). 
GMQE (Global Model Quality Estimation) is a quality estimation which combines properties from the target-template alignment and the template search method. QMEAN, which stands for Qualitative Model Energy Analysis, is a composite scoring function describing the major geometrical aspects of protein structures. All 3D models of the protein built by SWISS-MODEL server, were qualitatively estimated by GMQE and QMEAN scores (Schwede et al. 2003). In addition, $\mathrm{C}$-score is a confidence score for estimating the quality of predicted models by I-TASSER. It is calculated based on the significance of threading template alignments and the convergence parameters of the structure assembly simulations (Yang et al. 2015). Finally, Qualitative evaluation of 3D models was done by ProSA at https://prosa.servi ces.came.sbg.ac.at. ProSA specifically faces the needs confronted in the authentication of protein structures acquired from X-ray analysis, NMR spectroscopy, and hypothetical estimations (Wiederstein and Sippl 2007). Rampage at http://mordred.bioc.cam.ac.uk/rapper/rampage.php was also employed for estimation of model quality using Ramachandran plot which is an algorithm for atomic level, high-resolution protein structure improvement (Hooft et al. 1997).

\section{Molecular Docking}

The aim of molecular docking is to give a prediction of the binding affinity between a receptor molecule and ligand by computation methods (Ballester and Mitchell 2010). The vaccine construct $3(\mathrm{~V} 3)$ was made by I-TASSER server. In addition, PDB IDs of different HLA alleles were downloaded from the RCSB at https://www.rcsb.org/ (Rose et al. 2010). Molecular docking of V3 with 6 various HLA alleles including 1A6A(HLA-DR B1*03:01), 3C5J(HLA-DR B3*02:02), 1H15(HLA-DR B5*01:01), 2FSE(HLA-DRB1*01:01), 2Q6W(HLA-DR B3*01:01), and 2SEB(HLA-DRB1*04:01) was carried out by PatchDock server (Schneidman-Duhovny et al. 2005) at https://bioinfo3d.cs.tau.ac.il/PatchDock/ to represent HLA-peptide interactions. PatchDock predicted a number of 266 solutions that were again subjected to the FireDock server (http://bioinfo3d.cs.tau.ac.il/FireDock/) to refine and re-scoring of molecular docking score (Andrusier et al. 2007). TLRs are the most important membrane-bound PRRs, playing a critical role in the recognition of invading pathogens by the innate immune system. A. baumannii interacts with host cells mainly by engaging TLR 2 and TLR 4 (Chen 2020). In the same way, the PatchDock server was used to docking of vaccine construct (V3) with TLR2 (PDB ID 6NIG) and TLR4 (PDB ID 4G8A) and then the top 10 models were refined and re-scored by FireDock server. The docking models of TLRs with vaccine construct (V3) were displayed by the HADDOCK 2.4 server at https://wenmr. science.uu.nl/ (Van Zundert et al. 2016). The best cluster was chosen from the docked clusters based on the lowest HADDOCK score. Finally, the interacting residues, hydrogen bonds and hydrogen bonds distances between the vaccine and the TLRs were mapped using PDBsum at http:// www.ebi.ac.uk/thornton-srv/databases/cgi-bin/pdbsum/ GetPage.pl?pdbcode=index.html (Laskowski 2001).

\section{Prediction of Various Physicochemical Properties}

The functionally characterize of vaccine sequence was predicted by Expasy ProtParam server (https://web.expasy.org/ protparam/). This server has determined the physicochemical characteristics such as the molecular weight, number of amino acids, PI values, hydropathicity GRAVY values, instability index, aliphatic index, and estimated half-life of the protein. Calculate of these physicochemical properties is on the basis of $\mathrm{pK}$ values of different amino acids. The instability index value shows protein is stable or unstable. The instability index value is $<40$ for stable protein and $>40$ for unstable protein. The aliphatic index determines the volume occupied by the aliphatic side chains of protein (Gasteiger et al. 2005).

\section{Codon Optimization of the Vaccine Construct and In-Silico Cloning}

It is important to consider codon optimization when performing expression studies because codon optimization plays a critical role in the success of protein expression (Mauro and Chappell 2018). Java Codon Adaptation Tool (JCAT) at http://www.jcat.de/ was employed to adapt the codon usage of vaccine construct to $E$. coli host strain 12 . Vaccine amino acid sequence was back-translated to DNA sequence and then it was adapted for codon usage to E. coli. The adaptation was based on Codon Adaptation Index values (CAI). The rho-independent transcription terminators, prokaryotic ribosome binding sites, and cleavage sites of some restriction enzymes were avoided during the operation performed by JCAT server (Grote et al. 2005). In addition, to clone the adapted gene sequence of the final vaccine construct (V3) in E. coli pET28a vector (Ali et al. 2021), using Snapgene tool at https://www.snapgene.com/ to ensure the vaccine construct expression (Arumugam 2021) and BglII and ApaI restriction sites were introduced to the $\mathrm{N}$ and C-terminals of the sequence, respectively.

\section{Result}

The flow chart of experimental process which summarizes an overview of the methodology used in this study is shown in Fig. 1. 
Fig. 1 Flow chart showing an overview of our methodology
Fig. $2 \mathrm{CarO}$ sequence alignment with 20 sequences obtained from protein BLAST against Acinetobacter. The superposition was made with the T-coffee program and adjusted manually. Residues conservancy is depicted by blue to pink colors

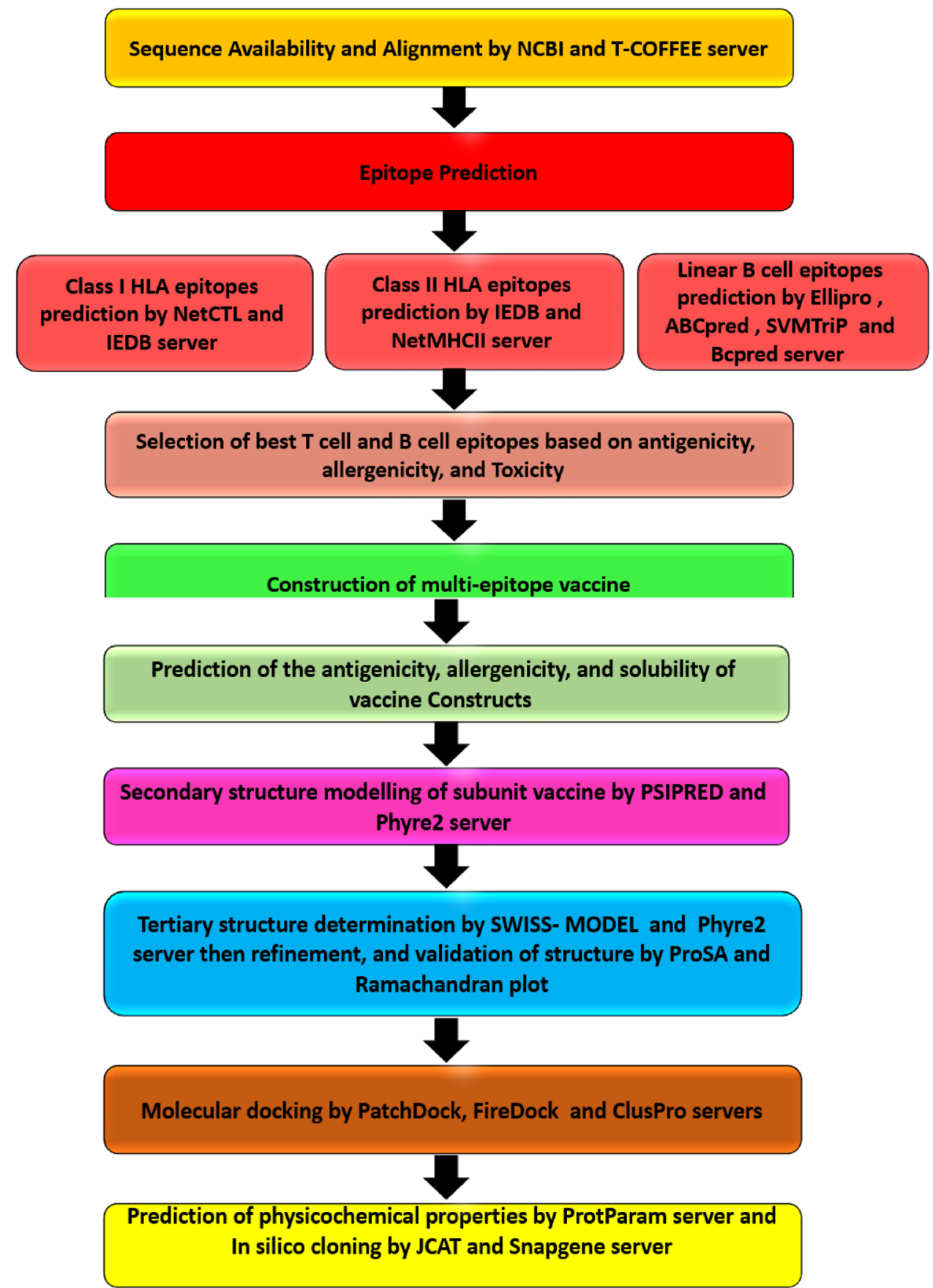

BAD AVG GOOD

AKL79738.1: 98

cons $\quad * * * . * \ldots *: * * *: * * * * *: * * .: * * * * * * * * * . * * * * * * * * * * * * * * * * * * * * * * *$

AKL79738.1 MKVLRVLVTTTALLAAGAAMADEAVVHDSYAFDKNQLIPVGARAEVGTTGYGGALLWQANPYVGLA

cons $\quad * * * * * * * * * * * *: . . * * . . * * * * * *$ : .***********************..*:*..*.*

AKL79738.1 LGYNGGDISWTDDVSVNGTKYOLDMDNNNVYLNAEIRPWGASTNPWAOGLYIAAGAAYLDNDYDLA

cons $\quad: \quad \ldots:: . \quad: \quad: \quad: *: . * *::: * *: * * * * * * *: * * * * * * * * * * * *: *$

AKL79738.1 KRIGNGDTLSIDGKNYQQAVPGQEG-GVRGKMSYKNDIAPYLGFGFAPKISKNWGVFGEVGAYYTG

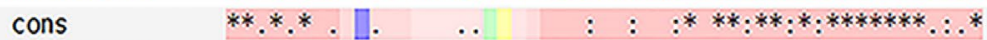

AKL79738.1 NPKVELTOY-NLAPVTGNPTSAQDAVDKEANEIRNDNKYEVMIPVGKVGVNFYW 
Table 1 CTL epitopes (MHC I peptides) of $\mathrm{CarO}$ protein with their antigenicity, allergenicity and immunogenicity scores

\begin{tabular}{|c|c|c|c|c|c|c|}
\hline No & CTL epitope & Position & Vaxijen score & Allergenicity & Immunogenicity & Final decision \\
\hline 1 & GAAYLDNDY & 121 & -0.6097 & Allergen & 0.02656 & \\
\hline 2 & RAEVGTTGY & 43 & 1.4273 & Allergen & 0.19614 & \\
\hline 3 & DVSVNGTKY & 79 & 1.7446 & Non allergen & -0.0798 & \\
\hline 4 & LLWQANPYV & 55 & 0.8355 & Allergen & -0.02417 & \\
\hline 5 & YIAAGAAYL & 117 & 0.1448 & Allergen & 0.15276 & \\
\hline 6 & YLDNDYDLA & 124 & -0.0090 & Allergen & 0.03105 & \\
\hline 7 & VLVTTTALL & 6 & -0.3603 & Allergen & 0.15334 & \\
\hline 8 & GVRGKMSYK & 158 & 2.1964 & Non allergen & -0.46618 & \\
\hline 9 & YLGFGFAPK & 172 & 1.0001 & Allergen & 0.29854 & \\
\hline 10 & AYLDNDYDL & 123 & -0.0481 & Allergen & 0.04314 & \\
\hline 11 & SYAFDKNQL & 29 & -0.0618 & Allergen & -0.12404 & \\
\hline 12 & IAPYLGFGF & 169 & 1.4363 & Non allergen & 0.13238 & $*$ \\
\hline 13 & GVFGEVGAY & 186 & -0.2548 & Allergen & 0.25992 & \\
\hline 14 & DVSVNGTKY & 79 & 1.7446 & Non allergen & -0.0798 & \\
\hline 15 & YIAAGAAYL & 117 & 0.1448 & Allergen & 0.15276 & \\
\hline 16 & SYKNDIAPY & 164 & 0.0616 & Allergen & 0.09691 & \\
\hline 17 & RVLVTTTAL & 5 & -0.3508 & Allergen & 0.1679 & \\
\hline 18 & IPVGARAEV & 38 & 0.3971 & Allergen & 0.22584 & \\
\hline 19 & NPTSAQDAV & 214 & 1.0699 & Allergen & -0.18323 & \\
\hline 20 & RVLVTTTAL & 5 & -0.3508 & Allergen & 0.1679 & \\
\hline 21 & KRIGNGDTL & 133 & 1.1551 & Non allergen & 0.1443 & $*$ \\
\hline 22 & YTGNPKVEL & 195 & 0.8255 & Non allergen & -0.11597 & \\
\hline 23 & YKNDIAPYL & 165 & -0.0820 & Allergen & 0.17513 & \\
\hline 24 & YIAAGAAYL & 117 & 0.1448 & Allergen & 0.15276 & \\
\hline 26 & DMDNNNVYL & 90 & 0.3279 & Allergen & 0.02098 & \\
\hline 27 & SYAFDKNQL & 29 & -0.0618 & Allergen & -0.12404 & \\
\hline 28 & WQANPYVGL & 57 & 0.8396 & Non allergen & 0.0466 & $*$ \\
\hline 29 & TGYGGALLW & 49 & 0.1673 & Allergen & 0.08689 & \\
\hline 30 & GKVGVNFYW & 241 & 1.6442 & Allergen & 0.17825 & \\
\hline 31 & YLNAEIRPW & 97 & 0.4980 & Allergen & 0.29725 & \\
\hline 32 & FAPKISKNW & 177 & 0.9830 & Allergen & -0.43251 & \\
\hline 33 & GVFGEVGAY & 186 & -0.2548 & Allergen & 0.25992 & \\
\hline 34 & ALLWQANPY & 54 & 0.6467 & Non allergen & 0.13138 & $*$ \\
\hline 35 & LYIAAGAAY & 116 & 0.4268 & Allergen & 0.20845 & \\
\hline 36 & AVVHDSYAF & 24 & 0.2032 & Allergen & -0.06844 & \\
\hline 37 & YLNAEIRPW & 97 & 0.4980 & Allergen & 0.29725 & \\
\hline 38 & YAFDKNQLI & 30 & -0.8295 & Allergen & -0.26001 & \\
\hline 39 & DEAVVHDSY & 22 & 0.5088 & Allergen & 0.04695 & \\
\hline 40 & YAFDKNQLI & 30 & -0.8295 & Allergen & -0.26001 & \\
\hline 41 & STNPWAQGL & 108 & -0.0570 & Allergen & 0.16131 & \\
\hline 42 & DMDNNNVYL & 90 & 0.3279 & Allergen & 0.02098 & \\
\hline 43 & AYYTGNPKV & 193 & 0.5286 & Allergen & -0.07059 & \\
\hline 44 & GVRGKMSYK & 158 & 2.1964 & No allergen & -0.46618 & \\
\hline 45 & VFGEVGAYY & 187 & -0.3389 & No allergen & 0.21471 & \\
\hline
\end{tabular}

Four CTL epitopes were selected (labeled with *) for incorporation in the vaccine construct

\section{Protein Sequencing and Alignments}

CarO protein sequence with accession No. AKL79738.1 is available as a query for protein BLAST against
Acinetobacter producing a set of sequences containing various species of Acinetobacter. Out of 100 alignments, 20 sequences with the highest similarity (identity $\geq 90 \%$, query coverage: $100 \%$ and $\mathrm{E}$ value: 0 ) were selected as T-COFFEE 
Table 2 HTL epitopes (MHC-II peptides) of $\mathrm{CarO}$ protein with their antigenicity, allergenicity, toxicity and mutation scores.

\begin{tabular}{|c|c|c|c|c|c|c|c|}
\hline No & HTL epitope & Position & Vaxijen Score & Allergenicity & Toxicity & Mutation & Final decision \\
\hline 1 & KVLRVLVTTTALLAA & $16-2$ & -0.2328 & No allergen & & & \\
\hline 2 & LRVLVTTTALLAAGA & $18-4$ & -0.0644 & No allergen & & & \\
\hline 3 & MKVLRVLVTTTALLA & $15-1$ & -0.1848 & No allergen & & & \\
\hline 4 & VLRVLVTTTALLAAG & $17-3$ & -0.2461 & No allergen & & & \\
\hline 5 & GNGDTLSIDGKNYQQ & $150-131$ & 1.8936 & No allergen & & & $*$ \\
\hline 6 & LSIDGKNYQQAVPGQ & $155-141$ & 1.1512 & Allergen & & & \\
\hline 7 & TLSIDGKNYQQAVPG & $154-140$ & 1.1523 & Allergen & & & \\
\hline 8 & PWAQGLYIAAGAAYL & $125-111$ & 0.5259 & No allergen & & & $*$ \\
\hline 9 & WAQGLYIAAGAAYLD & $126-112$ & 0.4335 & No allergen & & & $*$ \\
\hline 10 & AQGLYIAAGAAYLDN & $127-113$ & 0.4818 & No allergen & & & $*$ \\
\hline 11 & GLYIAAGAAYLDNDY & $129-115$ & 0.4596 & No allergen & & & $*$ \\
\hline 12 & QGLYIAAGAAYLDND & $128-114$ & 0.4293 & No allergen & & & $*$ \\
\hline 13 & ANEIRNDNKYEWMPV & $241-226$ & 1.7000 & No allergen & & & $*$ \\
\hline 14 & GKMSYKNDIAPYLGF & $175-161$ & 0.5176 & No allergen & & & $*$ \\
\hline 15 & KMSYKNDIAPYLGFG & $176-162$ & 0.4967 & No allergen & & & $*$ \\
\hline 16 & RGKMSYKNDIAPYLG & $174-160$ & 0.5852 & No allergen & & & $*$ \\
\hline 17 & VHDSYAFDKNQLIPV & $40-26$ & -0.1666 & No allergen & & & \\
\hline 18 & VRGKMSYKNDIAPYL & $173-159$ & 0.7463 & Allergen & & & \\
\hline 19 & IRPWGASTNPWAQGL & $102-116$ & -0.1476 & No allergen & & & \\
\hline 20 & DIAPYLGFGFAPKIS & $168-182$ & 1.2750 & No allergen & & & $*$ \\
\hline 21 & TALLAAGAAMADEAV & $11-25$ & -0.0024 & No allergen & & & \\
\hline 22 & NPWAQGLYIAAGAAY & $110-124$ & 0.1204 & No allergen & & & \\
\hline 23 & PWAQGLYIAAGAAYL & $111-125$ & 0.0259 & No allergen & & & \\
\hline 24 & VHDSYAFDKNQLIPV & $26-40$ & -0.1666 & Allergen & & & \\
\hline 25 & VLRVLVTTTALLAAG & $3-17$ & -0.2461 & Allergen & & & \\
\hline 26 & KVLRVLVTTTALLAA & $2-16$ & -0.2328 & Allergen & & & \\
\hline 27 & IAAGAAYLDNDYDLA & $118-132$ & -0.0341 & No allergen & & & \\
\hline 28 & NPWAQGLYIAAGAAY & $110-124$ & 0.1204 & No allergen & & & \\
\hline 29 & TALLAAGAAMADEAV & $11-25$ & -0.0024 & No allergen & & & \\
\hline 30 & VLVTTTALLAAGAAM & $6-20$ & -0.0596 & No allergen & & & \\
\hline 31 & AAGAAYLDNDYDLAK & $119-133$ & -0.0505 & No allergen & & & \\
\hline 32 & LVTTTALLAAGAAMA & $7-21$ & 0.0283 & Allergen & & & \\
\hline 33 & YLGFGFAPKISKNWG & $172-186$ & 1.7184 & No allergen & & & $*$ \\
\hline
\end{tabular}

Eleven HTL epitopes were selected (labeled with *) for selective step to incorporation in the vaccine construct

alignment input. BLAST search of Acinetobacter revealed numerous sequences of $\mathrm{CarO}$. These hits showed that the $\mathrm{CarO}$ protein is specific for Acinetobacter species. The image of PRALINE alignment results with conservation color scheme is shown in Fig. 2.

\section{Selection of Potent MHC-I Epitopes in Protein and Analysis Eminent Features of Epitopes}

On the basis of the high combinatorial score, the best T-cell epitopes were predicted by NetCTL server using the protein sequence. The software identified 36 epitopes in $\mathrm{CarO}$ protein. Peptides with combinatorial score $>1$ were selected to other analyses (Table 1). In IEDB server, 9 epitopes were elicited with both $\mathrm{IC}<200 \mathrm{nM}$ and percentile rank $\leq 0.2$ were subjected to afterward analysis (Table 1). After finalizing selected epitopes of both NetCTL and IEDB based above conditions, their important features including allergenicity, antigenicity, and immunogenicity Checked (Table 1). The sequences of IAPYLGFGF, KRIGNGDTL, WQANPYVGL and ALLWQANPY were considered as epitopes of T cells that interact with MHC Class-I and have the most important epitopes due to their features such as Non-allergen, positive scores in immunogenicity, and high scores in antigenicity. 
Fig. 3 Epitope mapping on 3D models. Discovery Studio Visualizer 2.5.5 software was used. From 1 to 6 pictures, 6 linear epitopes with the highest PI score predicted by Ellipro server are shown
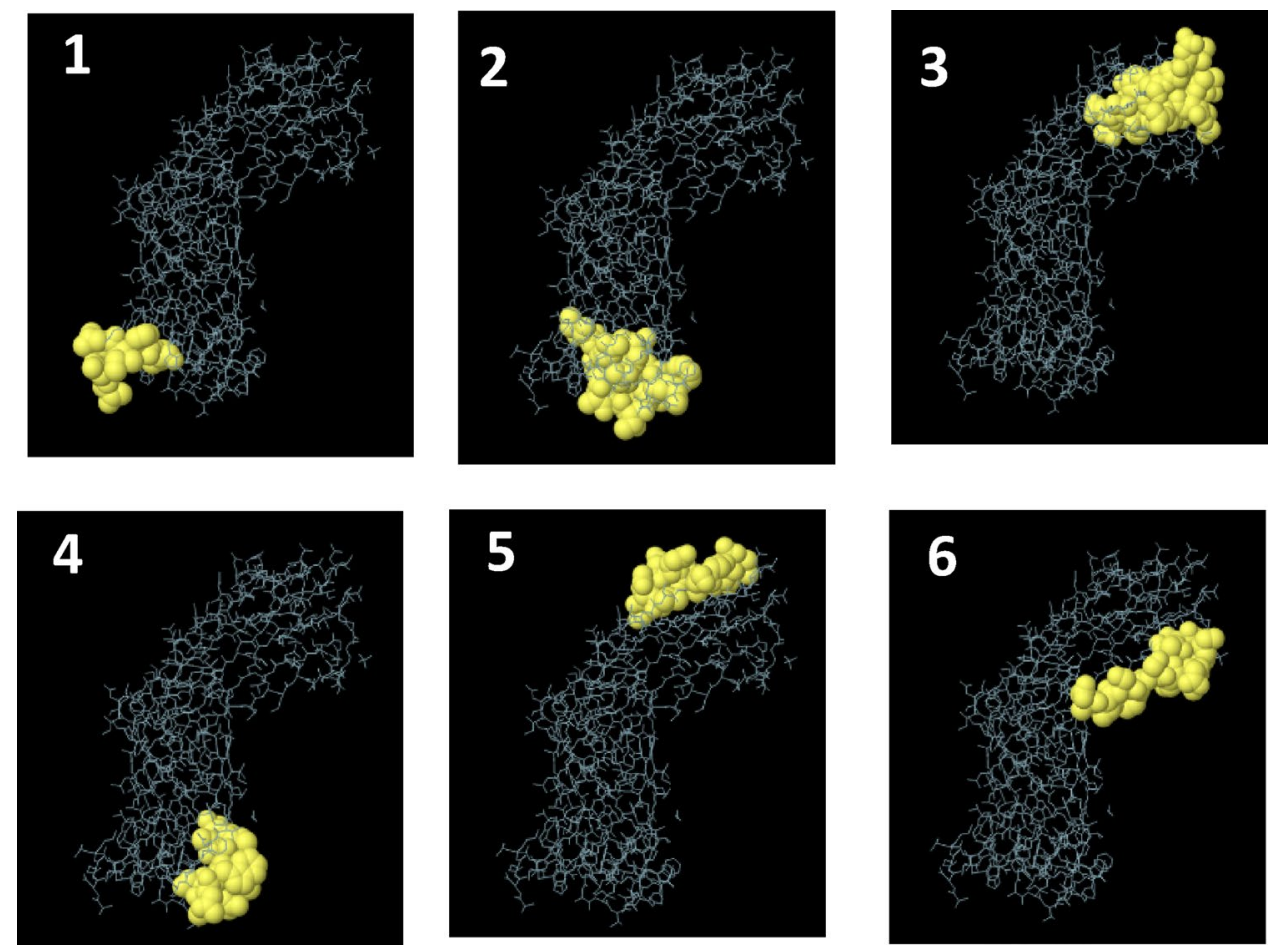

Table 3 B-cell epitopes of $\mathrm{CarO}$ protein with their antigenicity and allergenicity scores

\begin{tabular}{llllll}
\hline No & Bcell epitope & Position & Vaxijen Score & Allergenicity & Final decision \\
\hline 1 & SIDGKNYQQAVPGQEGGVRG & $142-161$ & 1.6824 & No allergen & $*$ \\
2 & LNAEIRPWGASTNPWAQGLY & $98-117$ & 0.1589 & Allergen & \\
3 & KNWGVFGEVGAYYTGNPKVE & $183-202$ & 0.0053 & Allergen & \\
4 & GYNGGDISWTDDVSVNGTKY & $68-87$ & 0.9330 & No allergen & $*$ \\
5 & APVTGNPTSAQDAVDKEANE & $209-228$ & 1.1717 & No allergen & $*$ \\
6 & NQLIPVGARAEVGTTGYGGA & $35-54$ & 0.8647 & No allergen & $*$ \\
7 & KNQLIPV & $34-40$ & -0.7431 & Allergen & \\
8 & IRPWGASTNRWAQGL & $81-95$ & 0.3082 & Allergen & \\
9 & NVDATRSFRVNNQDFIAGADGV & $113-134$ & 0.8684 & No allergen & $*$ \\
10 & FAPKINKNWGV & $154-164$ & 0.7523 & No allergen & $*$ \\
11 & SGSAVTTGDQSLEEAVNA & $183-200$ & 1.2565 & No allergen & $*$ \\
12 & DISWSDDVKVNGST & $52-65$ & 1.2709 & No allergen & $*$ \\
13 & AMADEAVVHDSYAFDKNQLI & $19-38$ & 0.0036 & Allergen & \\
14 & GAAYLDNDYDLAKRIGNGDT & $121-140$ & 0.3189 & Allergen & \\
15 & WQANPYVGLALGYNGGDISW & $57-76$ & 0.4059 & Allergen & \\
16 & ACINETACTERAMANN & $15-30$ & 0.5248 & No allergen & $*$ \\
17 & EVGAYYTGNPKVELTQ & $232-247$ & 0.5643 & No allergen & $*$ \\
18 & RAEVGTTGYGGALLWQ & $85-100$ & 0.9620 & No allergen & $*$ \\
19 & EAVVHDSYAFDKNQLI & $65-80$ & -0.1973 & Allergen & \\
20 & GGDISWTDDVSVNGTK & $113-228$ & 1.3248 & No allergen & $*$ \\
21 & DNKYEWMPVGKVGVNF & $274-289$ & 0.1697 & Allergen & \\
22 & PVTGNPTSAQDAVDKE & $252-267$ & 1.1236 & No allergen & $*$ \\
23 & TQYNLAPVTGNPTSAQ & $246-261$ & 0.8548 & Allergen & \\
24 & GQEGGVRGKMSYKNDI & $196-211$ & 1.9167 & Allergen & \\
\hline & & & & \\
& & &
\end{tabular}

Thirteen B-cell epitopes were selected (labeled with *) for selective step to incorporation in the vaccine construct 


\section{Selection of MHC-II Epitopes and Prediction Their Antigenic and Toxicity}

In addition to MHC-I epitope prediction, $\mathrm{CarO}$ protein was subjected to MHC-II binding prediction, using IEDB and NetMHCII server. The epitopes that elicited higher affinity $(\mathrm{IC}<200 \mathrm{nM})$ and percentile rank $\leq 0.2$ were subjected to afterward analysis (Table 2). NetMHCII server predicted more than 200 regions that could have an affinity for MHCII proteins. We selected 17 epitopes because these regions showed strong binding with a score $<1$ and promising characteristics. After this process, selected epitopes from both servers were analyzed based on their essential features including mutation, toxicity, allergenicity, and antigenicity (Table 2). Finally, the sequences of GNGDTLSIDGKNYQQ, PWAQGLYIAAGAAYL, WAQGLYIAAGAAY LD, AQGLYIAAGAAYLDN, GLYIAAGAAYLDNDY, QGLYIAAGAAYLDND, ANEIRNDNKYEWMPV, GKMSYKNDIAPYLGF, KMSYKNDIAPYLGFG, RGKMSYKNDIAPYLG, DIAPYLGFGFAPKIS, and YLGFGFAPKISKNWG as the epitopes of $T$ cells that interact with MHC Class-II alleles were considered as the most essential epitopes due to features of Non-allergen, non-toxin and high score in antigenicity.

\section{Linear B Cell Epitope Prediction}

The analysis of the sequences by different bioinformatics programs for $\mathrm{T}$ and $\mathrm{B}$ cells yielded approximately 100 epitopes. Svmtrip predicted 3 Linear B cell epitopes based on the score. The epitopes recommended by this server are "AMADEAVVHDSYAFDKNQLI", "GAAYLDNDYDLAKRIGNGDT", and "WQANPYVGLA LGYNGGDISW". 6 linear along B cell epitopes were predicted by ElliPro software. The sequences and pictures of 6 linear epitopes with the highest PI (protrusion index) are shown in Fig. 3 and Table 3 (Rows 7-12).

ABCpred result showed 65 hits of 16 metric peptide sequences as B-cell epitopes ranking based on scores. The best epitopes predicted by the server with a score above 0.85 are the sequences of "ACINETACTERAMANN", "EVGAYYTGNPKVELTQ", "RAEVGTTGYG GALLWQ", "EAVVHDSYAFDKNQLI", "GGDISWTDD VSVNGTK", "DNKYEWMPVGKVGVNF", "PVTGNPTSAQDAVDKE", "TQYNLAPVTGNPTSAQ", and "GQEGGVRGKMSYKNDI". BCpred server predicted 6 B-cell epitopes. The scores of epitopes ranging from 0.787 to 0.994 where a high score represents the higher probability of becoming B-cell epitopes and higher affinity
Table 4 The final selection of CTL, HTL and B-cell epitopes for multi-epitope vaccine construct

\begin{tabular}{llll}
\hline No & Type of epitope & Sequence of epitope & Antigenicity score \\
\hline 1 & CTL & IAPYLGFGF & 1.4363 \\
2 & CTL & KRIGNGDTL & 1.1551 \\
3 & CTL & WQANPYVGL & 0.8396 \\
4 & CTL & ALLWQANPY & 0.6467 \\
5 & HTL & GNGDTLSIDGKNYQQ & 1.8936 \\
6 & HTL & ANEIRNDNKYEWMPV & 1.7000 \\
7 & HTL & DIAPYLGFGFAPKIS & 1.2750 \\
8 & HTL & YLGFGFAPKISKNWG & 1.7184 \\
9 & B-cell & SIDGKNYQQAVPGQEGGVRG & 1.6824 \\
10 & B-cell & DISWSDDVKVNGST & 1.2709 \\
11 & B-cell & GGDISWTDDVSVNGTK & 1.3248 \\
12 & B-cell & PVTGNPTSAQDAVDKE & 1.1236 \\
\hline
\end{tabular}

Fig. 4 Schematic diagram of multi-epitope vaccine

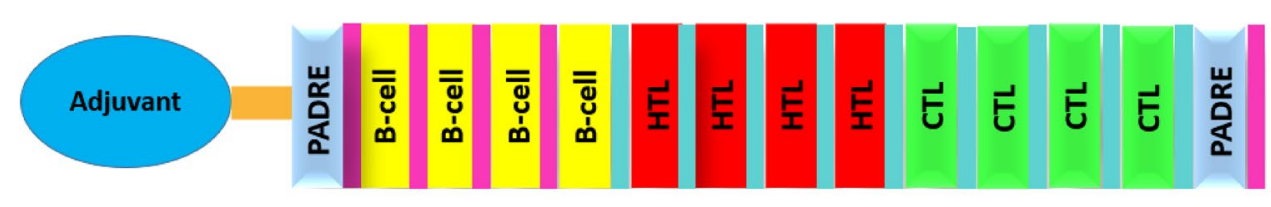

EAAAK linker

GGGS Linker

HEYGAEALERAG Linker 
Table 5 Allergenicity prediction of all vaccine construct using Algpred, Vaxijen and scratch protein predictor server

\begin{tabular}{|c|c|c|c|c|c|c|}
\hline No & $\begin{array}{l}\text { Vaccine } \\
\text { construct }\end{array}$ & Complete sequence of vaccine construct & $\begin{array}{l}\text { Allergenicity (Algpred) } \\
\text { (Threshold } \\
-0.4)\end{array}$ & $\begin{array}{l}\text { Vaxijen } \\
\text { Score }\end{array}$ & SOLpro & ANTIGENpro \\
\hline 1 & $\mathrm{~V}_{1}$ & $\begin{array}{l}\text { EAAAKMAKLSTDELLDAFKEMTLLELS- } \\
\text { DFVKKFEETFEVTAAAPVAVAAAGAA } \\
\text { PAGAAVEAAEEQSEFDVILEAAGDK- } \\
\text { KIGVIKVVREIVSGLGLKEAKDLVDGAP- } \\
\text { KPLLEKVAKEAADEAKAKLEAAGAT- } \\
\text { VTVKEAAAKAKFVAAWTLKAAAGGG } \\
\text { SSIDGKNYQQAVPGQEGGVRGGGGSDISWS- } \\
\text { DDVKVNGSTGGGSGGDISWTDDVSVNGT- } \\
\text { KGGGSPVTGNPTSAQDAVDKEHEYGAEALER- } \\
\text { AGGNGDTLSIDGKNYQQHEYGAEALERAGA- } \\
\text { NEIRNDNKYEWMPVHEYGAEALERAGDIAPYL- } \\
\text { GFGFAPKISHEYGAEALERAGYLGFGFAPKISKN- } \\
\text { WGHEYGAEALERAGIAPYLGFGFHEYGAEALER- } \\
\text { AGKRIGNGDTLHEYGAEALERAGWQANPYVGL- } \\
\text { HEYGAEALERAGALLWQANPYHEYGAEALERAG } \\
\text { AKFVAAWTLKAAAGGGS }\end{array}$ & $\begin{array}{l}0.22954849 \\
\text { Allergen }\end{array}$ & 0.8945 & 0.954742 & 0.831728 \\
\hline 2 & $\mathrm{~V}_{2}$ & $\begin{array}{l}\text { EAAAKGIINTLQKYYCRVRGGRCAVLSCLPKEEQI- } \\
\text { GKCSTRGRKCCRRKKEAAAKAKFVAAWTLKAAA } \\
\text { GGGSSIDGKNYQQAVPGQEGGVRGGGGSDIS- } \\
\text { WSDDVKVNGSTGGGSGGDISWTDDVSVNGT- } \\
\text { KGGGSPVTGNPTSAQDAVDKEHEYGAEALER- } \\
\text { AGGNGDTLSIDGKNYQQHEYGAEALERAGA- } \\
\text { NEIRNDNKYEWMPVHEYGAEALERAGDIAPYL- } \\
\text { GFGFAPKISHEYGAEALERAGYLGFGFAPKISKN- } \\
\text { WGHEYGAEALERAGIAPYLGFGFHEYGAEALER- } \\
\text { AGKRIGNGDTLHEYGAEALERAGWQANPYVGL- } \\
\text { HEYGAEALERAGALLWQANPYHEYGAEALERAG } \\
\text { AKFVAAWTLKAAAGGGS }\end{array}$ & $\begin{array}{l}-0.3428515 \\
\text { Allergen }\end{array}$ & 1.0459 & 0.972937 & 0.875245 \\
\hline 3 & $\mathrm{~V}_{3}$ & $\begin{array}{l}\text { EAAAKMAENPNIDDLPAPLLAALGAADLALAT- } \\
\text { VNDLIANLRERAEETRAETRTRVEERRARLTK- } \\
\text { FQEDLPEQFIELRDKFTTEELRKAAEGYLEAAT- } \\
\text { NRYNELVERGEAALQRLRSQTAFEDASARA- } \\
\text { EGYVDQAVELTQEALGTVASQTRAVGER- } \\
\text { AAKLVGIELEAAAKAKFVAAWTLKAAAGGG } \\
\text { SSIDGKNYQQAVPGQEGGVRGGGGSDISWS- } \\
\text { DDVKVNGSTGGGSGGDISWTDDVSVNGT- } \\
\text { KGGGSPVTGNPTSAQDAVDKEHEYGAEALER- } \\
\text { AGGNGDTLSIDGKNYQQHEYGAEALERAGA- } \\
\text { NEIRNDNKYEWMPVHEYGAEALERAGDIAPYL- } \\
\text { GFGFAPKISHEYGAEALERAGYLGFGFAPKISKN- } \\
\text { WGHEYGAEALERAGIAPYLGFGFHEYGAEALER- } \\
\text { AGKRIGNGDTLHEYGAEALERAGWQANPYVGL- } \\
\text { HEYGAEALERAGALLWQANPYHEYGAEALERA- } \\
\text { GAKFVAAWTLKAAAGGGS }\end{array}$ & $\begin{array}{l}-0.40767406 \\
\text { No allergen }\end{array}$ & 0.9251 & 0.950830 & 0.847375 \\
\hline
\end{tabular}


Table 5 (continued)

\begin{tabular}{|c|c|c|c|c|c|c|}
\hline No & $\begin{array}{l}\text { Vaccine } \\
\text { construct }\end{array}$ & Complete sequence of vaccine construct & $\begin{array}{l}\text { Allergenicity (Algpred) } \\
\text { (Threshold } \\
-0.4)\end{array}$ & $\begin{array}{l}\text { Vaxijen } \\
\text { Score }\end{array}$ & SOLpro & ANTIGENpro \\
\hline$\overline{4}$ & $\mathrm{~V}_{4}$ & $\begin{array}{l}\text { EAAAKMAENSNIDDIKAPLLAALGAADLA- } \\
\text { LATVNELITNLRERAEETRRSRVEESRARLT- } \\
\text { KLQEDLPEQLTELREKFTAEELRKAAE- } \\
\text { GYLEAATSELVERGEAALERLRSQQSFEEVS- } \\
\text { ARAEGYVDQAVELTQEALGTVASQVEGRAA- } \\
\text { KLVGIELEAAAKAKFVAAWTLKAAAGGG } \\
\text { SSIDGKNYQQAVPGQEGGVRGGGGSDISWS- } \\
\text { DDVKVNGSTGGGSGGDISWTDDVSVNGT- } \\
\text { KGGGSPVTGNPTSAQDAVDKEHEYGAEALER- } \\
\text { AGGNGDTLSIDGKNYQQHEYGAEALERAGA- } \\
\text { NEIRNDNKYEWMPVHEYGAEALERAGDIAPYL- } \\
\text { GFGFAPKISHEYGAEALERAGYLGFGFAPKISKN- } \\
\text { WGHEYGAEALERAGIAPYLGFGFHEYGAEALER- } \\
\text { AGKRIGNGDTLHEYGAEALERAGWQANPYVGL- } \\
\text { HEYGAEALERAGALLWQANPYHEYGAEALERAG } \\
\text { AKFVAAWTLKAAAGGGS }\end{array}$ & $\begin{array}{l}-0.2874046 \\
\text { Allergen }\end{array}$ & 0.9330 & 0.966516 & 0.819423 \\
\hline
\end{tabular}

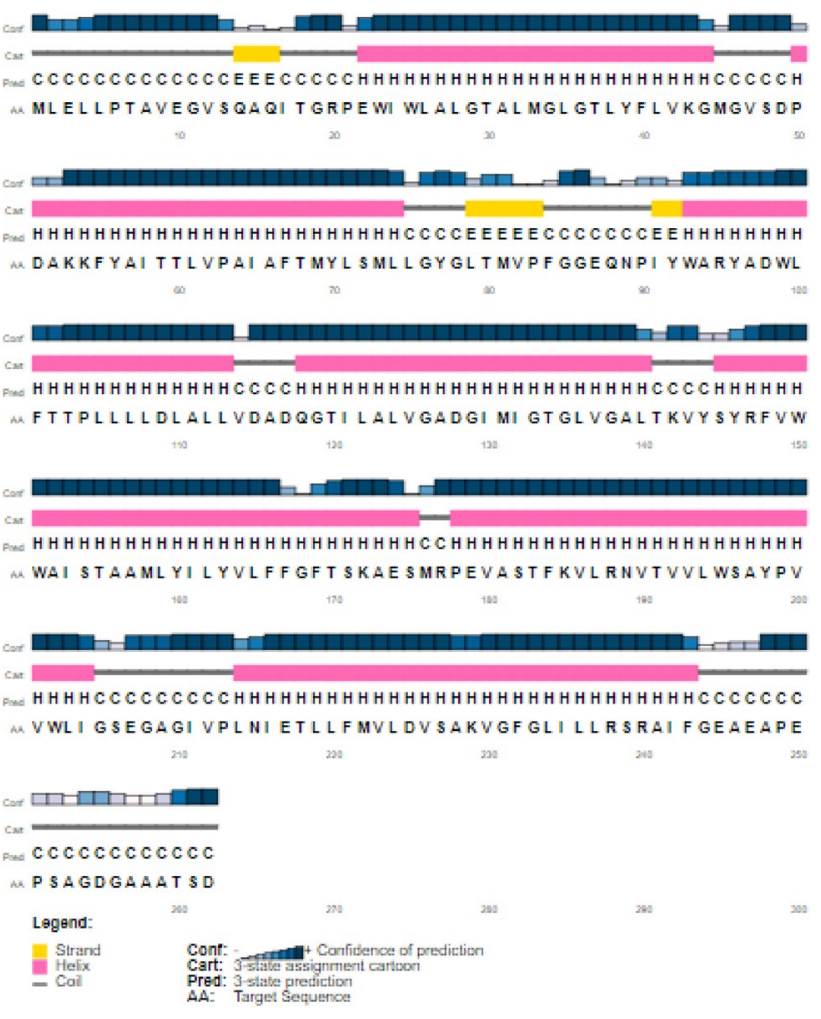

Fig. 5 Secondary structure prediction of vaccine construct (V3) using PESIPRED server

for the B-cell receptor. The total promising linear B cell epitopes which were mentioned above were analyzed by the Vaxijen server ( $\geq 0.4$ score) and AllergenFP server in order to participate in the vaccine construct.

\section{Comparison of All the Predicted Epitopes to Select the Final Epitopes}

After collecting the immunoinformatics information with the help of T-cell prediction and B-cell prediction, the high scores of HTL, CTL, and B cell epitopes were predicted and analyzed for their essential feature. Finally, to make the final multi-epitope construct, 4 CTL epitopes, 4 HTL epitopes, and $4 \mathrm{~B}$ cell epitopes due to features of non-Allergen, nontoxic, and higher score in antigenicity (Table 4).

\section{Construction of Final Vaccine with Different Adjuvants, Linkers, and PADRE Sequence}

A total of four vaccine constructs were prepared and further analyzed. All four vaccine constructs were added with the respective adjuvants with the help of EAAAK linker. MHCI, MHC-II, B-epitopes, and PADRE sequence were joined together by HEYGAEALERAG and GGGS linkers (Fig. 4). Details of the vaccine constructs have been mentioned in Table 5.

\section{Prediction Features of Different Vaccine Constructs}

Antigenicity of the vaccine proteins was predicted using VaxiJen server and ANTIGENpro. All Constructs were found as potential vaccine candidates with high antigenicity scores and the ability to stimulate an efficient immune response (Table 5). Also all 4 constructs showed the solubility more than 0.7 , which indicated that the vaccine construct will be highly soluble during its heterologous expression in the E. coli (Table 5). But, among four constructs, the only 


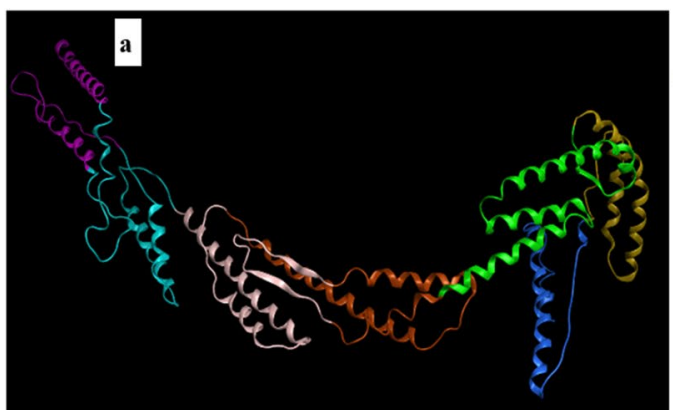

Fig. 6 a The best model of epitope vaccine (V3) predicted by I-TASSER server. $\mathbf{b}$ All of the regions of the vaccine for display on the scaffold are labeled and colored in representing adjuvants_yellow,

vaccine with beta-defensin $\left(\mathrm{V}_{3}\right)$ was found to be non-allergic in nature (Allergenicity $<-0.4$ ). So, the $\mathrm{V}_{3}$ construct was chosen for further analysis.

\section{Prediction Secondary Structure}

PSIPRED and Phyre2 servers were used to predict the secondary structure of the construct V3. The predicted structure of the protein confirmed to have $68.70 \%$ alpha-helix, $27.48 \%$ sheet, and $3.82 \%$ coil structure (Fig. 5). The results from Phyre 2 confirmed the results of the PSIPRED program.

\section{Vaccine 3D Modeling, Refinement, and Validation}

Swiss model and I-TASSER software were recruited for homology modeling and $\mathrm{Ab}$ initio prediction. The former prepared 12 different models and the latter introduced 5 models. Among the structures predicted by the Swiss model, the best structures were selected based on the QMEAN and GMQE scores. QMEAN scores about zero show good agreement between the model structure and experimental structures of similar size and scores of -4.0 or below indicate models with low quality. In addition, the GMQE score is expressed as a number between

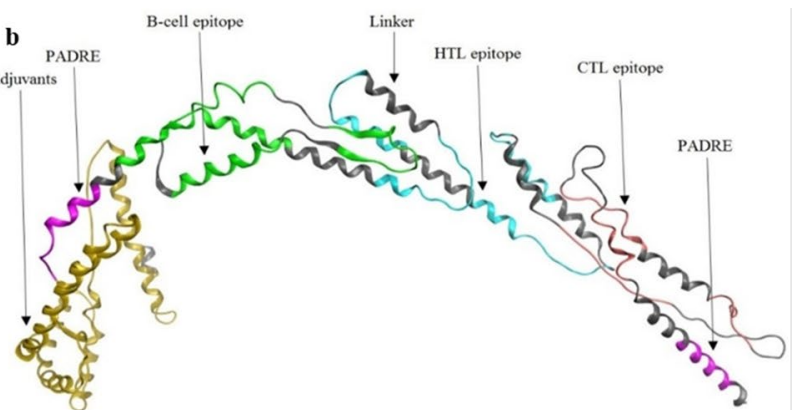

PADRE_purple, B-cell epitopes_green, Linker_gray, HTL_blue, and CTL_pink (Color figure online)

zero and one, reflecting the expected accuracy of a model built with that alignment and template. Higher numbers illustrate higher reliability. Two 3D models have shown GMQE with scores -1.41 and -1.38 and QMEAN with scores of 0.05 and 0.04 respectively. The best structure among five models of I-TASSER were measured on the basis of $\mathrm{C}$-score. The $\mathrm{C}$-score is normally in $[-5,2]$ and It should be noted that the higher this score, the more accurate the model quality. Since the $\mathrm{C}$-score of model 2 is higher $(=-0.83)$ than other models, is expected to have good quality. Finally, three predicted 3D models were selected for further analyses.

The quality of the 3D models were evaluated by Rampage and Prosa servers. Based on the result of Ramachandran plot and Prosa the best model was chosen from the predicted structure by I-TASSER (Fig. 6a). We showed the Cartoon diagram of a computational structural model of the vaccine in the following figure by using Molecular Operating Environment Software. This figure displays that the sequence of each epitope is properly folded and exposed (Fig. 6b). This structure of vaccine construct show which $87.438 \%$ of the residues are in favored regions. Also, Prosa revealed that the predicted model was among other acceptable proteins with z score $=-5.27$ (Fig. 7).

Table 6 Prediction binding energy of vaccine construct (V3) with selected HLA molecules by PatchDock, Firedock

\begin{tabular}{|c|c|c|c|c|c|c|}
\hline $\begin{array}{l}\text { Vaccine } \\
\text { constructs }\end{array}$ & $\begin{array}{l}\text { HLA alleles \& their } \\
\text { PDB ID's }\end{array}$ & Score & Area & $\begin{array}{l}\text { Hydrogen } \\
\text { bond energy }\end{array}$ & $\begin{array}{l}\text { Globel } \\
\text { energy }\end{array}$ & $\mathrm{ACE}$ \\
\hline \multirow[t]{6}{*}{$\mathrm{V} 3$} & HLA-DRB1*03:01 (1A6A) & 13,384 & 2181.40 & -1.41 & 0.33 & 3.51 \\
\hline & HLA-DR B3*02:02 (3C5J) & 14,840 & 2191.30 & -4.17 & -10.18 & 12.89 \\
\hline & HLA-DR B5*01:01 (1H15) & 14,816 & 2309.50 & -1.23 & -14.97 & 1.11 \\
\hline & HLA-DR B1*01:01 (2FSE) & 13,982 & 1947.70 & -4.11 & -21.21 & 8.95 \\
\hline & HLA-DRB3*01:01 (2Q6W) & 15,688 & 2164.50 & -0.23 & -3.22 & 2.43 \\
\hline & HLA-DR B1*04:01 (2SEB) & 13,990 & 1956.80 & -0.44 & -5.23 & 0.36 \\
\hline
\end{tabular}


a

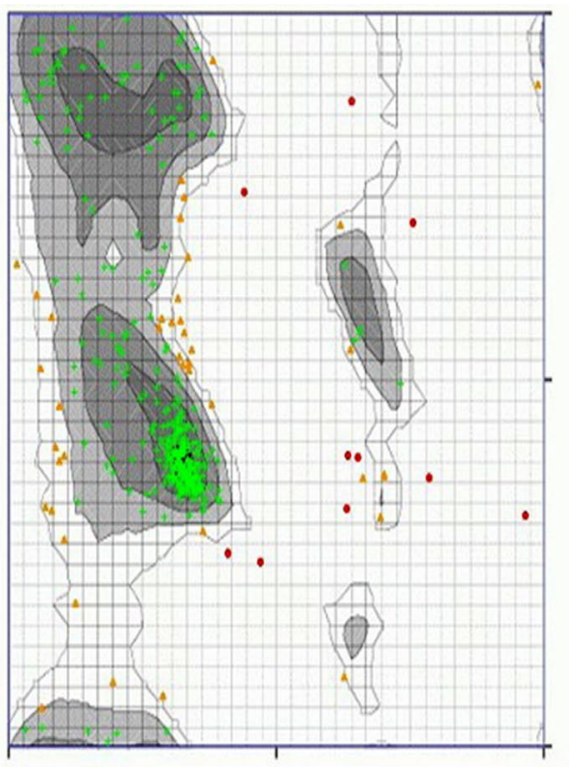

The chart is color-coded for your convenience:

Black Dark Grey Grey Light Grey represent Highly Preferred Conformations. Delta $>=-2$

White with Black Grid represents preferred conformations. $-2>$ Delta $>=-4$

White with Grey Grid represents questionable conformations. Delta $<-4$

Highly Preferred observartions shown as GREEN Crosses: 355 (87.438\%)

Preferred observations shown as BROWN Triangles: 41 (10.099\%)

Ouestronable observations shown as RED Curcles 10 (2.463\%)
Z-Score: $-5.27 \quad \mathbf{b}$

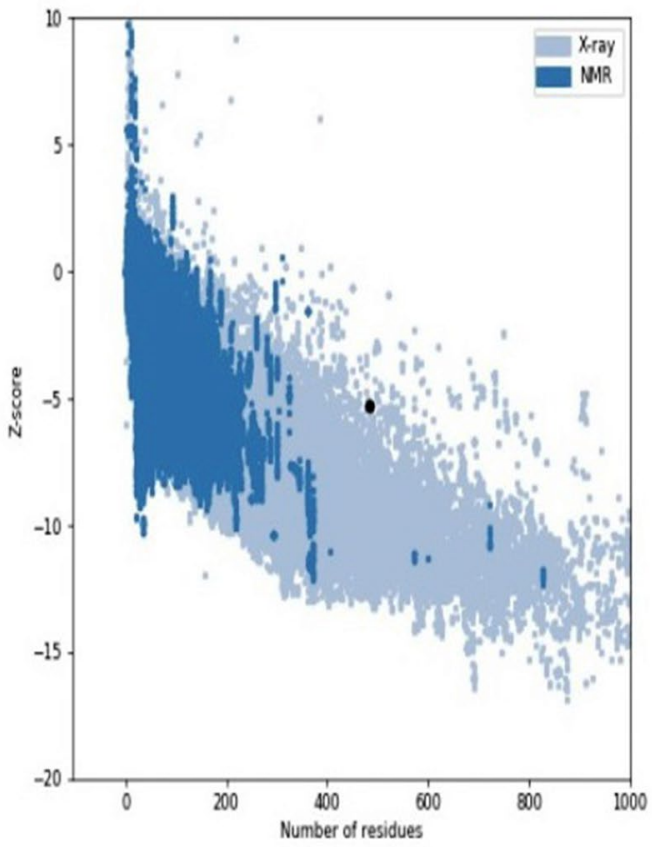

Number of residues in outlier region: $10(2.463 \%)$. b Prosa protein structure analysis results. $\mathrm{Z}$ score $=-5.27$. Overall quality of the ultimate model is acceptable

hydrogen bonds and distance of hydrogen bonds are shown in Figs. 8 and 9 and Tables 7 and 8.

\section{Physicochemical Analysis of Vaccine Construct (V3)}

The vaccine construct (V3) was characterized on the basis of various physicochemical properties using ProtParam tool. The molecular weight of the vaccine construct was found $51.57 \mathrm{kDa}$ which shows its good antigenic potential. GRAVY (a hydropathic index) was -0.527 (a negative value), which represents the hydrophilic nature of constructs. The calculated instability index of the protein was 23.43 which classified it as a stable one. The estimated half-life of the constructed vaccine was expected to be $1 \mathrm{~h}$ in mammalian reticulocytes in vitro while more than $10 \mathrm{~h}$ in E. coli in vivo. Aliphatic value 71.90 also showed that the vaccine construct has a good characteristic to initiate an immunogenic reaction. 


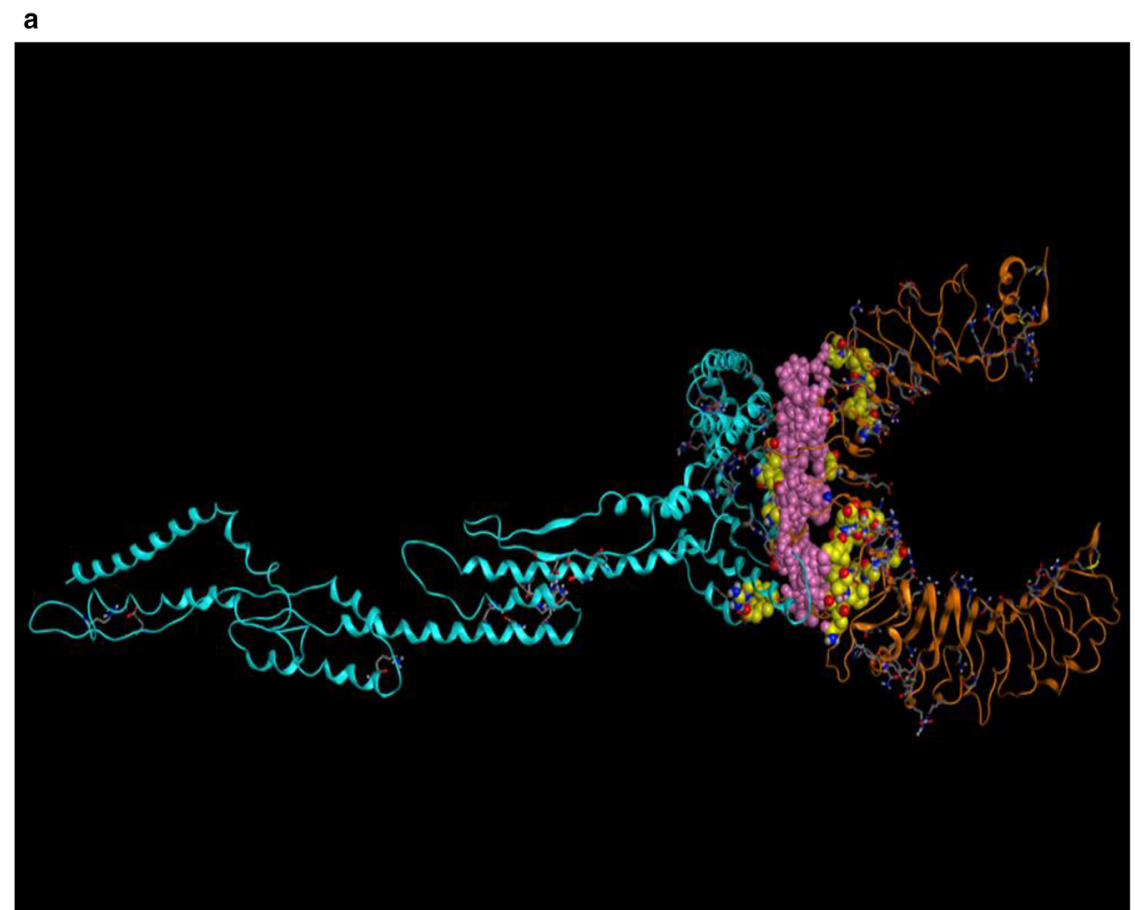

b

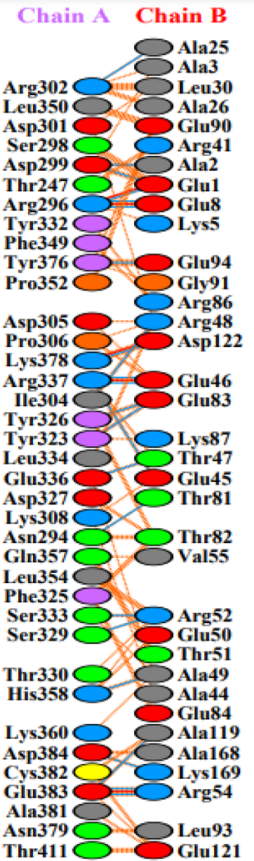

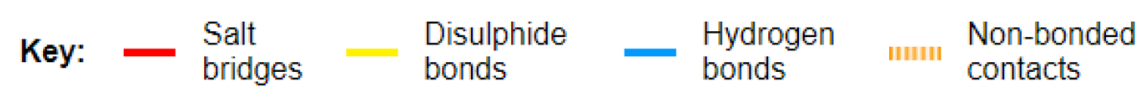

Fig. 8 a Showing TLR2/vaccine docked complex. Vaccine construct 3 is shown in blue colour while TLR2 is shown in orange colour. Interacting residues and active pockets between docked TLR2/vac-

\section{In-Silico Cloning of Chimeric Vaccine Construct (V3) for Its Heterologous Expression}

Reverse translation and codon optimization were performed by considering the expression system of the host in order to express the multi-epitope vaccine. The optimum range of GC content was considered to be between 30 and $70 \%$. The GC content of the optimized codons (53.95\%) was significant. Also, the high value of the CAI score shows a high expression of the gene. Our construct has a codon adaptation index $(\mathrm{CAI})$ of 1 . Recognition sites for BgIII and ApaI restriction enzymes were added to the $5^{\prime}$ and $3^{\prime}$ end of the optimized gene and then the final vaccine construct (V3) with a total length of $1465 \mathrm{bp}$ was cloned into pET28a vector for its heterologous cloning and expression in E. coli (Fig. 10). Polyhistidine-tag is an amino acid motif in proteins that typically consists of at least six histidines (His) residues which is often at the $\mathrm{N}$ - or C-terminus with spaced or attached to inserted protein. His-tag will be used to purify protein in further experiments. In addition, the His-tag is part of the pET28a vector structure. cine are displayed with purple and yellow filling space model respectively. b Few prominent hydrogen bonds within vaccine-TLR2 complex are focused (Color figure online)

\section{Discussion}

The emergence of multidrug resistance (MDR) A. baumannii is recognized as a serious health concern in hospitals across the world (Savov et al. 2019). CarO from A. baumannii is a membrane porin that uptake small molecules such as L-ornithine, Carbapenem, and other basic amino acids (Labrador-Herrera et al. 2020; Tohidinia et al. 2020). In a study, researchers were demonstrated outer membrane vesicles (OMVs) of A. baumannii had able to induce protective immunity against $A$. baumannii. Among OMV proteins, $\mathrm{Car} O$ was identified as a highly immunogenic protein (McConnell et al. 2011). Currently, there is no definite cure to protect against the A. baumannii due to increase MDR. Therefore, it is essential to take preventive measures against it (Solanki and Tiwari 2018). The employment of bioinformatics tools in the field of vaccinology can be aiding the selection of potential vaccine candidates and simplify the optimization of the chosen immunogen (Gupta and Kumar 2020; Tohidinia and Sefid 2020). 


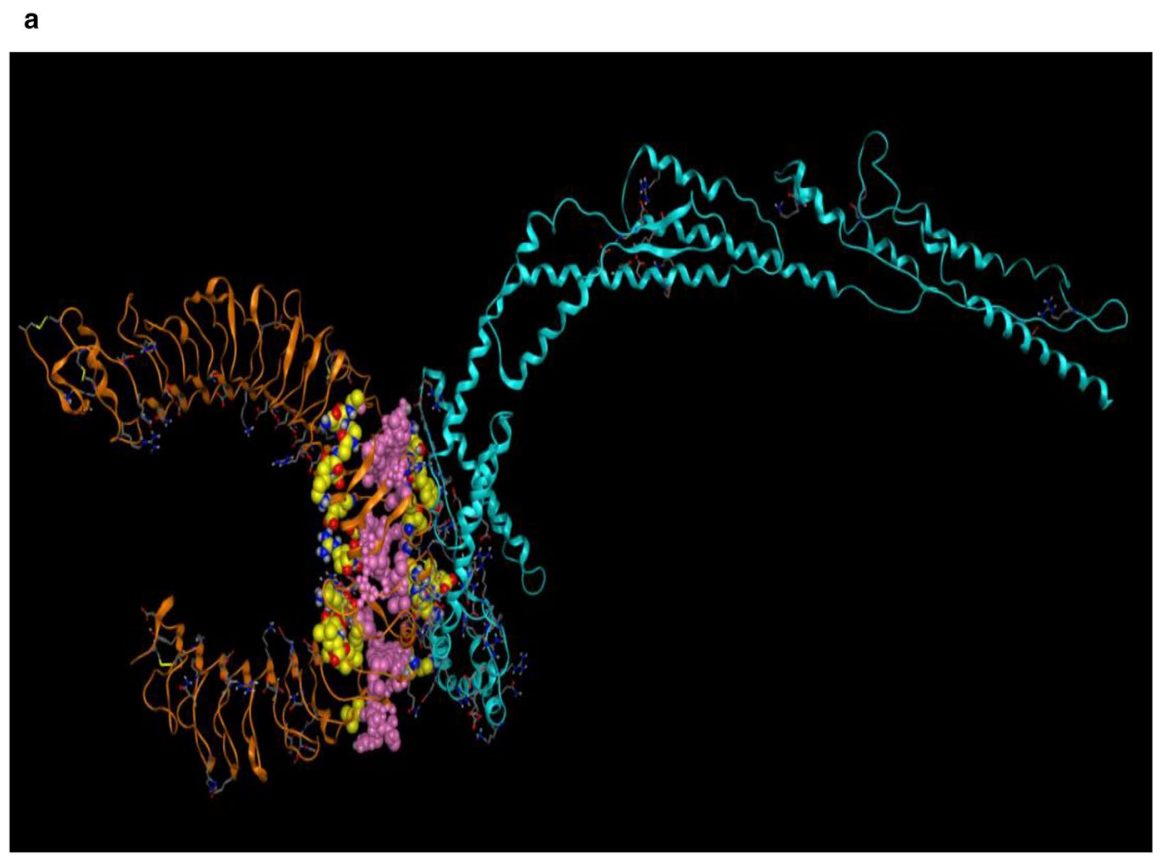

b

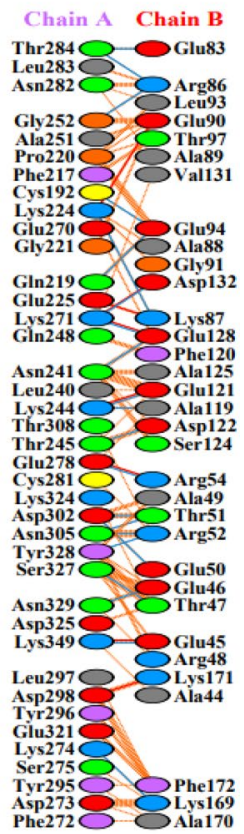

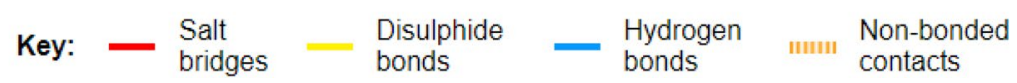

Fig. 9 a Showing TLR4/vaccine docked complex. Vaccine construct 3 is shown in blue colour while TLR4 is shown in orange colour. Interacting residues and active pockets between docked TLR4/vac-

The entire proteome of the A. baumannii ATCC 19606 strain was retrieved from NCBI and the result of BLAST from various sequences of $\mathrm{CarO}$ showed it is specific to Acinetobacter species. Alignment of $\mathrm{CarO}$ sequences from various strains indicates that such vaccines are able to trigger antibodies with complete specificity for $A$. baumannii and $\mathrm{CarO}$ is a conserved protein among Acinetobacter species. Therefore, it can one of the best choices for vaccine design. A multi-epitope vaccine consists of several $\mathrm{B}$ and $\mathrm{T}$ epitopes with sensible linkers in order to avoid the generation of junctional epitopes and increase the antigen presentation process (Forouharmehr 2021; Shey et al. 2019). A multi-epitope vaccine can trigger both humoral and cell-mediated immunity (Ali et al. 2017). The cell-mediated immune response is mediated by T-cells whereas the humoral immune response is mediated by produced B-cells (Channappanavar et al. 2014; Kozakiewicz et al. 2013). Therefore, the correct and accurate selection of TCL, HTL, and B-cell epitopes to design a multi-epitope vaccine has a direct relationship with the immune system response.

Approximately 300 epitopes of $\mathrm{CarO}$ protein were predicted by IEDB with recommended methods and NetCTL server to be MHC I peptides that can bind to a large number of HLA-A and HLA-B alleles with a high binding affinity. 350 epitopes of $\mathrm{CarO}$ protein were also predicted cine are displayed with purple and yellow filling space model respectively. b Few prominent hydrogen bonds within vaccine-TLR4 complex are focused (Color figure online)

by using the MHC-II binding prediction tool of IEDB and NetMHCII server as MHC II peptides. But the most important epitopes were selected among the identified epitopes with certain scores mentioned in the method section and then their important characteristics were examined. One of the most important features considered for selecting CTL epitopes was the Vaxijen score. The higher Vaxijen score indicating the higher antigenicity of an amino acid sequence and is able to further stimulate the immune system (Saha et al. 2017). In this study, only CTL epitopes with non-allergenic and positive immunogenicity behavior were selected for further analysis. In addition, the characteristics of the allergenicity, antigenicity, toxicity, and mutation were examined for the selection of HTL epitopes. Finally, according to all these characteristics, sequences IAPYLGFGF, KRIGNGDTL, WQANPYVGL, and ALLWQANPY as CTL epitopes and GNGDTLSIDGKNYQQ, ANEIRNDNKYEWMPV, DIAPYLGFGFAPKIS, and YLGFGFAPKISKNWG as HTL epitopes were selected to participate in the structure of the vaccine. Amino acid sequences that are linear in shape are called continuous or linear epitopes (Abboud et al. 2009) which were predicted by ElliPro, ABCpred, Svmtrip, and BCpred servers. Through the epitope prediction servers carried out in this project we were able to compile 82 potential linear B cell 
Table 7 List of residues involved in hydrogen bonds and hydrogen bonds distance in TLR2-vaccine interactions

\begin{tabular}{|c|c|c|c|c|c|c|c|}
\hline No. & Res name & Res no. & Chain & Res name & Res no. & Chain & $\begin{array}{l}\text { Distance } \\
\text { hydrogen bonds }\end{array}$ \\
\hline 1 & ASN & 294 & A & THR & 81 & B & 3.03 \\
\hline 2 & ARG & 296 & A & GLU & 1 & B & 2.67 \\
\hline 3 & ARG & 296 & A & GLU & 8 & B & 2.75 \\
\hline 4 & ARG & 296 & A & GLU & 8 & B & 2.6 \\
\hline 5 & ASP & 299 & A & GLU & 1 & B & 2.75 \\
\hline 6 & ASP & 299 & A & ALA & 2 & B & 2.84 \\
\hline 7 & ARG & 302 & A & ALA & 25 & B & 2.79 \\
\hline 8 & ILE & 304 & A & ARG & 48 & B & 2.69 \\
\hline 9 & TYR & 323 & A & GLU & 83 & B & 2.63 \\
\hline 10 & TYR & 326 & A & GLU & 83 & B & 3.11 \\
\hline 11 & SER & 329 & A & ARG & 52 & B & 3.04 \\
\hline 12 & SER & 333 & A & ARG & 52 & B & 2.77 \\
\hline 13 & GLU & 336 & A & THR & 47 & B & 2.65 \\
\hline 14 & ARG & 337 & A & GLU & 45 & B & 2.97 \\
\hline 15 & ARG & 337 & A & GLU & 46 & B & 3 \\
\hline 16 & ARG & 337 & A & GLU & 46 & B & 2.78 \\
\hline 17 & HIS & 358 & A & ALA & 49 & B & 2.66 \\
\hline 18 & TYR & 376 & A & GLU & 94 & B & 2.61 \\
\hline 19 & LYS & 378 & A & ASP & 122 & B & 2.62 \\
\hline 20 & GLU & 383 & A & ALA & 119 & B & 3.1 \\
\hline 21 & GLU & 383 & A & ARG & 54 & B & 2.66 \\
\hline 22 & GLU & 383 & A & ARG & 54 & B & 3.06 \\
\hline 23 & ASP & 384 & A & LYS & 169 & B & 2.67 \\
\hline
\end{tabular}

Table 8 List of residues

involved in hydrogen bonds and hydrogen bonds distance in TLR4-vaccine interactions

\begin{tabular}{|c|c|c|c|c|c|c|c|}
\hline No. & Res name & Res no. & Chain & Res name & Res no. & Chain & $\begin{array}{l}\text { Distance } \\
\text { hydrogen bonds }\end{array}$ \\
\hline 1 & CYS & 192 & A & GLU & 94 & B & 2.98 \\
\hline 2 & GLN & 219 & A & GLU & 94 & B & 2.65 \\
\hline 3 & LYS & 224 & A & LYS & 87 & B & 2.67 \\
\hline 4 & ASN & 241 & A & GLU & 128 & B & 3.1 \\
\hline 5 & LYS & 244 & A & ALA & 119 & B & 2.79 \\
\hline 6 & LYS & 244 & A & GLU & 121 & B & 2.65 \\
\hline 7 & THR & 245 & A & ASP & 122 & B & 2.64 \\
\hline 8 & GLY & 252 & A & ARG & 86 & B & 2.86 \\
\hline 9 & LYS & 271 & A & GLU & 128 & B & 2.54 \\
\hline 10 & LYS & 271 & A & ASP & 132 & B & 2.71 \\
\hline 11 & LYS & 274 & A & LYS & 169 & B & 3.31 \\
\hline 12 & GLU & 278 & A & ARG & 54 & B & 2.57 \\
\hline 13 & THR & 284 & A & GLU & 83 & B & 3.05 \\
\hline 14 & THR & 284 & A & ARG & 86 & B & 2.84 \\
\hline 15 & ASP & 302 & A & GLU & 50 & B & 3 \\
\hline 16 & ASP & 302 & A & THR & 51 & B & 2.67 \\
\hline 17 & ASN & 305 & A & ARG & 52 & B & 2.7 \\
\hline 18 & ASN & 305 & A & THR & 51 & B & 2.78 \\
\hline 19 & SER & 327 & A & THR & 47 & B & 2.62 \\
\hline 20 & TYR & 328 & A & ARG & 52 & B & 3.04 \\
\hline 21 & ASN & 329 & A & GLU & 46 & B & 2.67 \\
\hline 22 & LYS & 349 & A & GLU & 45 & B & 2.65 \\
\hline
\end{tabular}


Fig. 10 In silico cloning of the gene sequence of the final vaccine construct V3 into pET28a (+) expression vector. The target sequence was inserted between BglII (401) and ApaI (1334)

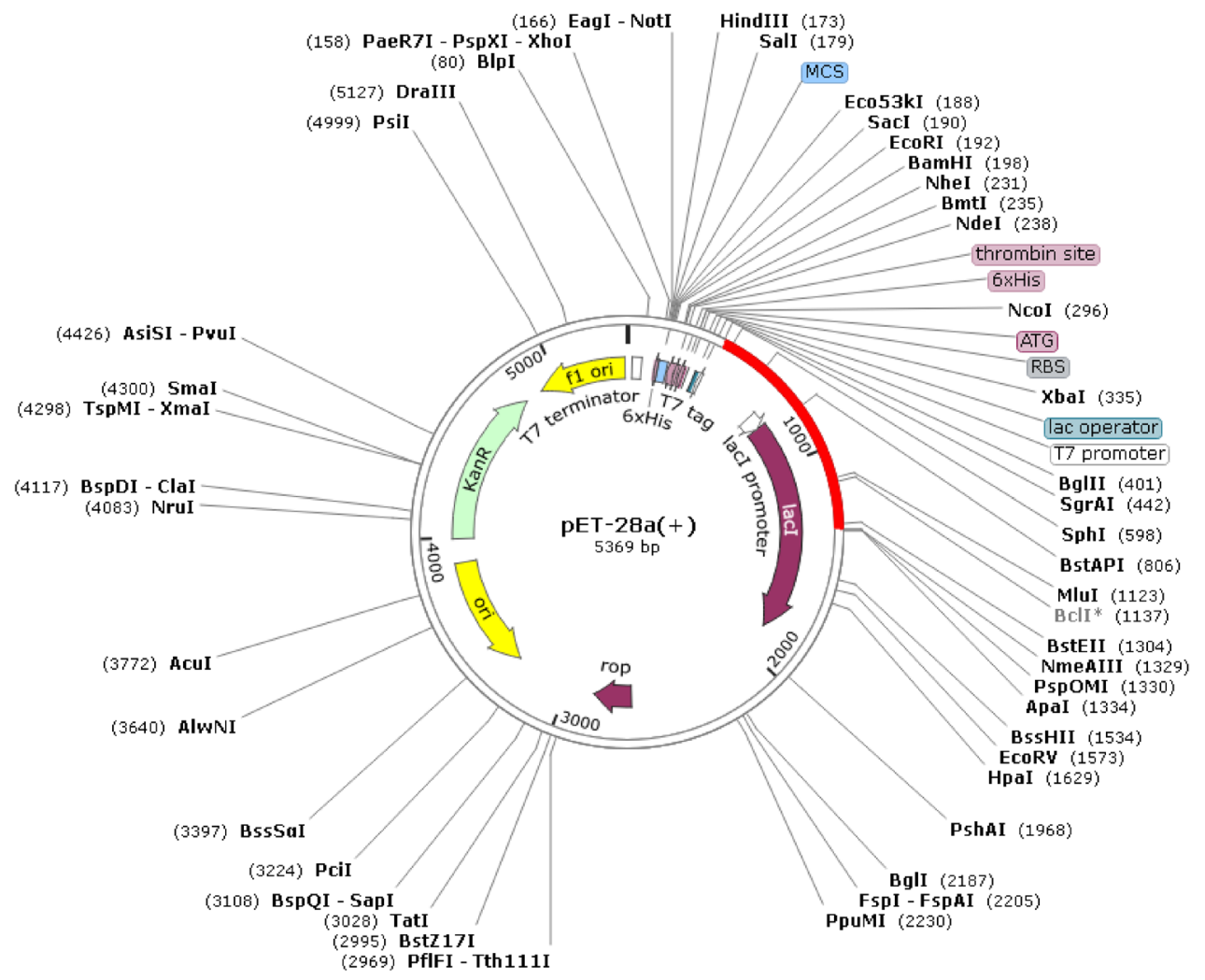

epitopes in $\mathrm{CarO}$ protein. Among promising linear B cell epitopes, four B cell epitopes were selected based on their features such as antigenicity and allergenicity.

In the construction of vaccines, the selected epitopes were connected by HEYGAEALERAG and GGGS linkers. Each of these constructions was connected to 4 different adjuvants with the EAAAK linker. Finally, after examining the allergenicity, antigenicity, and solubility behaviors vaccine construct 3 which had HBHA adjuvant was selected due to non-allergic in nature and high allergenicity and antigenicity score to prediction the second and third structures.

To ensure effective binding between HLA molecules and vaccine construct 3, a docking study was performed. The vaccine construct 3 was subjected to the PatchDock server as a receptor and 6 different HLA alleles including 1A6A(HLA-DR B1*03:01), 3C5J(HLA-DR B3*02:02), 1H15(HLA-DR B5*01:01), 2FSE(HLA-DRB1*01:01), 2Q6W(HLA-DR B3*01:01), and 2SEB(HLA-DRB1*04:01) as a ligand were selected for docking analysis. All the selected HLA showed low binding energy with V3 which was biologically significant. HLA-DR B1*01:01 was found to be best considering the free binding energy. Moreover, to display interactions among human TLRs (TLR2 and TLR4) and the vaccine structure 3, HADDOCK server were applied for molecular docking. Docking results demonstrated that the multi-epitope vaccine we designed has the potential to bind the mention receptors. Also, the PDBsum server showed 22 and 23 hydrogen bonds between the vaccine and TLR2 and TLR4 respectively. Hydrogen bonds can increase the strength and stability of a vaccine-receptor interaction substantially (Fox et al. 2013). Physicochemical properties of the V3 sequence were analyzed using ProtParam server which showed nature of V3 is appropriate for vaccine construct. Finally, the vaccine construct V3 was reverse transcribed and adapted for $E$. coli strain K12 former to insertion within pET28a (+) vector for its cloning and expression. In our study, our in silico results were based on various databases. We suggest using model animals for further analysis and experimental validation of this designed vaccine.

Acknowledgements We thank Yazd University for granting us a permission to use its web server.

Funding This study was supported by an unrestricted free access to Yazd University web site for data collection.

\section{Declarations}

Conflict of interest The authors have received no financial support for the elaboration of this manuscript. Yazd University did not play any decision-making role in the study analysis or writing of the manuscript. All authors declare no potential conflicts of interest.

Ethical Approval As the present study involved no experimental with animals or human, hence there was no need for approval by the Ethics Committee of Yazd University. 


\section{References}

Abboud $\mathrm{N}$ et al (2009) Identification of linear epitopes in Bacillus anthracis protective antigen bound by neutralizing antibodies. $\mathrm{J}$ Biol Chem 284:25077-25086

Ali M, Pandey RK, Khatoon N, Narula A, Mishra A, Prajapati VK (2017) Exploring dengue genome to construct a multi-epitope based subunit vaccine by utilizing immunoinformatics approach to battle against dengue infection. Sci Rep 7:1-13

Ali S et al (2021) Proteome wide vaccine targets prioritization and designing of antigenic vaccine candidate to trigger the host immune response against the Mycoplasma genitalium infection. Microb Pathog 152:104771

Anderson L, Dormitzer P, Nokes D, Rappuoli R, Roca A, Graham B (2013) Strategic priorities for respiratory syncytial virus (RSV) vaccine development. Vaccine 31:B209-B215

Andrusier N, Nussinov R, Wolfson HJ (2007) FireDock: fast interaction refinement in molecular docking. Proteins 69:139-159

Arumugam S (2021) In-silico design of envelope based multi-epitope vaccine candidate against Kyasanur forest disease virus. Sci Rep. https://doi.org/10.21203/rs.3.rs-155690/v1

Asif M, Alvi IA, Rehman SU (2018) Insight into Acinetobacter baumannii: pathogenesis, global resistance, mechanisms of resistance, treatment options, and alternative modalities. Infect Drug Resist 11:1249

Ayoub Moubareck C, Hammoudi Halat D (2020) Insights into Acinetobacter baumannii: a review of microbiological, virulence, and resistance traits in a threatening nosocomial pathogen. Antibiotics 9:119

Ballester PJ, Mitchell JB (2010) A machine learning approach to predicting protein-ligand binding affinity with applications to molecular docking. Bioinformatics 26:1169-1175

Behbahani M (2020) In silico design of novel multi-epitope recombinant vaccine based on coronavirus surface glycoprotein. Bioinformatics 17:122

Calis JJ et al (2013) Properties of MHC class I presented peptides that enhance immunogenicity. PLoS Comput Biol 9:e1003266

Catel-Ferreira M et al (2011) Structure-function relationships of $\mathrm{CarO}$, the carbapenem resistance-associated outer membrane protein of Acinetobacter baumannii. J Antimicrob Chemother 66:2053-2056

Cayatte C, Marin A, Rajani GM, Schneider-Ohrum K, Snell Bennett A, Marshall JD, Andrianov AK (2017) PCPP-adjuvanted respiratory syncytial virus (RSV) sF subunit vaccine: self-assembled supramolecular complexes enable enhanced immunogenicity and protection. Mol Pharm 14:2285-2293

Centers for Disease Control and Prevention (2019) 2019 AR threats report. https://www.cdc.gov/drugresistance/biggest-threats.html\# acine

Chandra S, Singh TR (2012) Linear B cell epitope prediction for epitope vaccine design against meningococcal disease and their computational validations through physicochemical properties. Netw Model Anal Health Inf Bioinform 1:153-159

Channappanavar R, Zhao J, Perlman S (2014) T cell-mediated immune response to respiratory coronaviruses. Immunol Res 59:118-128

Chen W (2020) Host innate immune responses to Acinetobacter baumannii infection. Front Cell Infect Microbiol 10:486

Dar A, Tipu M, Townsend H, Potter A, Gerdts V, Tikoo S (2015) Administration of poly [di (sodium carboxylatoethylphenoxy) phosphazene](PCEP) and avian beta defensin as adjuvants in inactivated inclusion body hepatitis virus and its hexon proteinbased experimental vaccine formulations in chickens. Avian Dis 59:518-524
Dijkshoorn L, Nemec A, Seifert H (2007) An increasing threat in hospitals: multidrug-resistant Acinetobacter baumannii. Nat Rev Microbiol 5:939-951

Dimitrov I, Naneva L, Doytchinova I, Bangov I (2014) AllergenFP: allergenicity prediction by descriptor fingerprints. Bioinformatics 30:846-851

Doener F et al (2019) RNA-based adjuvant CV8102 enhances the immunogenicity of a licensed rabies vaccine in a first-in-human trial. Vaccine 37:1819-1826

Dolan MA, Noah JW, Hurt D (2011) Comparison of common homology modeling algorithms: application of user-defined alignments. In: Homology modeling. Humana Press, Totowa, pp 399-414

Doytchinova IA, Flower DR (2007) VaxiJen: a server for prediction of protective antigens, tumour antigens and subunit vaccines. BMC Bioinform 8:4

Eijkelkamp BA, Stroeher UH, Hassan KA, Paulsen IT, Brown MH (2014) Comparative analysis of surface-exposed virulence factors of Acinetobacter baumannii. BMC Genomics 15:1-12

Eliopoulos GM, Maragakis LL, Perl TM (2008) Acinetobacter baumannii: epidemiology, antimicrobial resistance, and treatment options. Clin Infect Dis 46:1254-1263

EL-Manzalawy Y, Dobbs D, Honavar V (2008) Predicting linear B-cell epitopes using string kernels. J Mol Recognit 21:243-255

Espinal P et al (2019) Relationship between different resistance mechanisms and virulence in Acinetobacter baumannii. Microb Drug Resist 25:752-760

Forouharmehr A (2021) Engineering an efficient poly-epitope vaccine against Toxoplasma gondii infection: a computational vaccinology study. Microb Pathog 152:104646

Fox CB, Kramer RM, Barnes VL, Dowling QM, Vedvick TS (2013) Working together: interactions between vaccine antigens and adjuvants. Ther Adv Vaccines 1:7-20

Gasparini R, Montomoli E, Fragapane E, Senatore F, Minutello M, Podda A (2001) Increased immunogenicity of the MF59-adjuvanted influenza vaccine compared to a conventional subunit vaccine in elderly subjects. Eur J Epidemiol 17:135-140

Gasteiger E, Hoogland C, Gattiker A, Wilkins MR, Appel RD, Bairoch A (2005) Protein identification and analysis tools on the ExPASy server. In: Walker JM (ed) The proteomics protocols handbook. Humana Press, Totowa, pp 571-607

Grote A, Hiller K, Scheer M, Münch R, Nörtemann B, Hempel DC, Jahn D (2005) JCat: a novel tool to adapt codon usage of a target gene to its potential expression host. Nucleic Acids Res 33:W526-W531

Gupta N, Kumar A (2020) Designing an efficient multi-epitope vaccine against Campylobacter jejuni using immunoinformatics and reverse vaccinology approach. Microb Pathog 147:104398

Gupta S, Kapoor P, Chaudhary K, Gautam A, Kumar R, Raghava GP, Open Source Drug Discovery Consortium (2013) In silico approach for predicting toxicity of peptides and proteins. PLoS ONE 8:e73957

Gupta N, Regar H, Verma VK, Prusty D, Mishra A, Prajapati VK (2020) Receptor-ligand based molecular interaction to discover adjuvant for immune cell TLRs to develop next-generation vaccine. Int J Biol Macromol 152:535-545

Hasan M, Ghosh PP, Azim KF, Mukta S, Abir RA, Nahar J, Khan MMH (2019) Reverse vaccinology approach to design a novel multi-epitope subunit vaccine against avian influenza A (H7N9) virus. Microb Pathog 130:19-37

Higgins PG, Dammhayn C, Hackel M, Seifert H (2010) Global spread of carbapenem-resistant Acinetobacter baumannii. J Antimicrob Chemother 65:233-238 
Hooft RW, Sander C, Vriend G (1997) Objectively judging the quality of a protein structure from a Ramachandran plot. Bioinformatics 13:425-430

Howard A, O'Donoghue M, Feeney A, Sleator R (2012) Acinetobacter baumannii: an emerging opportunistic pathogen. Virulence 3:243-250

Jensen KK et al (2018) Improved methods for predicting peptide binding affinity to MHC class II molecules. Immunology 154:394-406

Kalita P, Padhi AK, Zhang KY, Tripathi T (2020) Design of a peptidebased subunit vaccine against novel coronavirus SARS-CoV-2. Microb Pathog 145:104236

Kaushik V, Therapeutics (2019) In silico identification of epitope-based peptide vaccine for Nipah virus. Int J Peptide Res Ther. https:// doi.org/10.1007/s10989-019-09917-0

Kazi A, Chuah C, Majeed ABA, Leow CH, Lim BH, Leow CY (2018) Current progress of immunoinformatics approach harnessed for cellular-and antibody-dependent vaccine design. Pathog Glob Health 112:123-131

Kelley LA, Mezulis S, Yates CM, Wass MN, Sternberg MJE (2015) The Phyre 2 web portal for protein modeling, prediction and analysis. Nat Protoc 10:845-858

Kiefer F, Arnold K, Künzli M, Bordoli L, Schwede T (2009) The SWISS-MODEL repository and associated resources. Nucleic Acids Res 37:D387-D392

Kozakiewicz L, Phuah J, Flynn J, Chan J (2013) The role of B cells and humoral immunity in Mycobacterium tuberculosis infection. In: Divangahi M (ed) The new paradigm of immunity to tuberculosis. Advances in experimental medicine and biology. Springer, New York, pp 225-250

Kumar S, Sunagar R, Gosselin E (2019) Bacterial protein toll-likereceptor agonists: a novel perspective on vaccine adjuvants. Front Immunol 10:1144

Kyriakidis I, Vasileiou E, Pana ZD, Tragiannidis A (2021) Acinetobacter baumannii antibiotic resistance mechanisms. Pathogens 10:373

Labrador-Herrera G et al (2020) Virulence role of the outer membrane protein CarO in carbapenem-resistant Acinetobacter baumannii. Virulence 11:1727-1737

Larsen MV, Lundegaard C, Lamberth K, Buus S, Brunak S, Lund O, Nielsen M (2005) An integrative approach to CTL epitope prediction: a combined algorithm integrating MHC class I binding, TAP transport efficiency, and proteasomal cleavage predictions. BMC Bioinform 35:2295-2303

Larsen MV, Lundegaard C, Lamberth K, Buus S, Lund O, Nielsen M (2007) Large-scale validation of methods for cytotoxic T-lymphocyte epitope prediction. BMC Bioinform 8:424

Laskowski RA (2001) PDBsum: summaries and analyses of PDB structures. Nucleic Acids Res 29:221-222

Lei Y, Shao J, Ma F, Lei C, Chang H, Zhang Y (2020) Enhanced efficacy of a multi-epitope vaccine for type $\mathrm{A}$ and $\mathrm{O}$ foot-and-mouth disease virus by fusing multiple epitopes with Mycobacterium tuberculosis heparin-binding hemagglutinin (HBHA), a novel TLR4 agonist. Mol Immunol 121:118-126

Magnan CN, Randall A, Baldi P (2009) SOLpro: accurate sequencebased prediction of protein solubility. Bioinformatics 25:2200-2207

Mallick A et al (2007) Escheriosome-mediated delivery of recombinant ribosomal L7/L12 protein confers protection against murine brucellosis. Vaccine 25:7873-7884

Mauro VP, Chappell SA (2018) Considerations in the use of codon optimization for recombinant protein expression. In: Recombinant protein expression in mammalian cells. Springer, New York, pp 275-288

McConnell MJ, Domínguez-Herrera J, Smani Y, López-Rojas R, Docobo-Pérez F, Pachón J (2011) Vaccination with outer membrane complexes elicits rapid protective immunity to multidrug-resistant Acinetobacter baumannii. Infect Immun 79:518-526

McGuffin LJ, Bryson K, Jones DT (2000) The PSIPRED protein structure prediction server. Bioinformatics 16:404-405

Mishra R, Krishan S, Siddiqui AN, Kapur P, Khayyam KU, Sharma M (2020) Potential role of adjuvant drugs on efficacy of first line oral antitubercular therapy: drug repurposing. Tuberculosis 120:101902

Mussi MA, Relling VM, Limansky AS, Viale AM (2007) CarO, an Acinetobacter baumannii outer membrane protein involved in carbapenem resistance, is essential for L-ornithine uptake. FEBS Lett 581:5573-5578

Nezafat N, Ghasemi Y, Javadi G, Khoshnoud MJ, Omidinia E (2014) A novel multi-epitope peptide vaccine against cancer: an in silico approach. J Theor Biol 349:121-134

Nguyen van Binh P, Duc HT (2006) Epitopes of the class I major histocompatibility complex (MHC-I) recognized in the syngeneic or allogeneic context predominantly linked to antigenic peptide loading to its binding groove. Clin Exp Immunol 145:372-379

Obeidat N, Jawdat F, Al-Bakri AG, Shehabi AA (2014) Major biologic characteristics of Acinetobacter baumannii isolates from hospital environmental and patients' respiratory tract sources. Am J Infect Control 42:401-404

Palatnik-de-Sousa C, Soares I, Rosa D (2018) Editorial: epitope discovery and synthetic vaccine design. Front Immunol 9:826

Perez F, Hujer AM, Hujer KM, Decker BK, Rather PN, Bonomo RA (2007) Global challenge of multidrug-resistant Acinetobacter baumannii. Antimicrob Agents Chemother 51:3471-3484

Poirel L, Nordmann P (2006) Carbapenem resistance in Acinetobacter baumannii: mechanisms and epidemiology. Clin Microbiol Infect $12: 826-836$

Ponomarenko J, Bui H-H, Li W, Fusseder N, Bourne PE, Sette A, Peters B (2008) ElliPro: a new structure-based tool for the prediction of antibody epitopes. BMC Bioinform 9:514

Pruitt KD, Tatusova T, Maglott DR (2005) NCBI Reference Sequence (RefSeq): a curated non-redundant sequence database of genomes, transcripts and proteins. Nucleic Acids Res 33:D501-D504

Rana A, Akhter Y (2016) A multi-subunit based, thermodynamically stable model vaccine using combined immunoinformatics and protein structure based approach. Immunobiology 221:544-557

Rashidian E, Forouharmehr A, Nazifi N, Jaydari A, Shams N (2020) Computer-aided design of a novel poly-epitope protein in fusion with an adjuvant as a vaccine candidate against leptospirosis. Curr Proteomics 17:1-11

Rasooli I, Abdolhamidi R, Jahangiri A, Astaneh SDA (2020) Outer membrane protein, Oma87 prevents Acinetobacter baumannii infection. Int J Pept Res Ther 26(4):2653-2660

Rose PW et al (2010) The RCSB Protein Data Bank: redesigned web site and web services. Nucleic Acids Res 39:D392-D401

Rubinstein ND, Mayrose I, Halperin D, Yekutieli D, Gershoni JM, Pupko T (2008) Computational characterization of B-cell epitopes. Mol Immunol 45:3477-3489

Saadi M, Karkhah A, Nouri HR (2017) Development of a multi-epitope peptide vaccine inducing robust $\mathrm{T}$ cell responses against brucellosis using immunoinformatics based approaches. Infect Genet Evol 51:227-234

Saha S, Raghava GPS (2006a) AlgPred: prediction of allergenic proteins and mapping of IgE epitopes. Nucleic Acids Res 34:W202-W209

Saha S, Raghava GPS (2006b) Prediction of continuous B-cell epitopes in an antigen using recurrent neural network. Proteins 65:40-48

Saha CK, Hasan MM, Hossain MS, Jahan MA, Azad AK (2017) In silico identification and characterization of common epitopebased peptide vaccine for Nipah and Hendra viruses. Asian Pac J Trop Med 10:529-538 
Savov E, Trifonova A, Kovachka K, Kjosseva E, Strateva T (2019) Antimicrobial in vitro activities of ceftazidime-avibactam, meropenem-vaborbactam and plazomicin against multidrug-resistant Acinetobacter baumannii and Pseudomonas aeruginosa - a pilot Bulgarian study. Infect Dis 51:870-873

Schneidman-Duhovny D, Inbar Y, Nussinov R, Wolfson HJ (2005) PatchDock and SymmDock: servers for rigid and symmetric docking. Nucleic Acids Res 33:W363-W367

Schwede T, Kopp J, Guex N, Peitsch MC (2003) SWISS-MODEL: an automated protein homology-modeling server. Nucleic Acids Res 31:3381-3385

Shaker MA, Shaaban MI (2017) Formulation of carbapenems loaded gold nanoparticles to combat multi-antibiotic bacterial resistance: in vitro antibacterial study. Int J Pharm 525:71-84

Shey RA et al (2019) In-silico design of a multi-epitope vaccine candidate against onchocerciasis and related filarial diseases. Sci Rep 9:1-18

Simossis VA, Heringa J (2003) The PRALINE online server: optimising progressive multiple alignment on the web. Elsevier, Amsterdam

Simossis VA, Heringa J (2005) PRALINE: a multiple sequence alignment toolbox that integrates homology-extended and secondary structure information. Comput Biol Chem 33:W289-W294

Siroy A et al (2005) Channel formation by CarO, the carbapenem resistance-associated outer membrane protein of Acinetobacter baumannii. Antimicrob Agents Chemother 49:4876-4883

Solanki V, Tiwari V (2018) Subtractive proteomics to identify novel drug targets and reverse vaccinology for the development of chimeric vaccine against Acinetobacter baumannii. Sci Rep 8:1-19

Suschak JJ, Williams JA, Schmaljohn CS (2017) Advancements in DNA vaccine vectors, non-mechanical delivery methods, and molecular adjuvants to increase immunogenicity. Hum Vaccines Immunother 13:2837-2848

Tatusova TA, Madden TL (1999) BLAST 2 sequences, a new tool for comparing protein and nucleotide sequences. FEMS Microbiol Lett 174:247-250

Tohidinia M, Sefid F (2020) Identification B and T-cell epitopes and functional exposed amino acids of $\mathrm{S}$ protein as a potential vaccine candidate against SARS-CoV-2/COVID-19. Microb Pathog 148:104459

Tohidinia M, Moshtaghioun SM, Sefid F, Falahati A (2020) Functional exposed amino acids of CarO analysis as a potential vaccine candidate in Acinetobacter Baumannii. Int J Pept Res Ther 26:1185-1197
Valencia R et al (2009) Nosocomial outbreak of infection with pandrug-resistant Acinetobacter baumannii in a tertiary care university hospital. Infect Control Hosp Epidemiol 30:257

Van Zundert G et al (2016) The HADDOCK2. 2 web server: userfriendly integrative modeling of biomolecular complexes. J Mol Biol 428:720-725

Vita $R$ et al (2019) The immune epitope database (IEDB): 2018 update. Nucleic Acids Res 47:D339-D343

Wang $M$ et al (2020) The improvement of immune effect of recombinant human beta-defensin 2 on hepatitis B vaccine in mice. Viral Immunol 34:96-111

Wheeler D (2003) Selecting the right protein-scoring matrix. Curr Protoc Bioinform. https://doi.org/10.1002/0471250953.bi0305s00

Wiederstein M, Sippl MJ (2007) ProSA-web: interactive web service for the recognition of errors in three-dimensional structures of proteins. Nucleic Acids Res 35:W407-W410

Wu C-Y, Monie A, Pang X, Hung C-F, Wu TC (2010) Improving therapeutic HPV peptide-based vaccine potency by enhancing CD4+ T help and dendritic cell activation. J Biomed Sci 17:1-10

Yang J, Zhang Y (2015) Protein structure and function prediction using I-TASSER. Curr Protoc Bioinform. https://doi.org/10.1002/ 0471250953.bi0508s52

Yang J, Yan R, Roy A, Xu D, Poisson J, Zhang Y (2015) The I-TASSER Suite: protein structure and function prediction. Nat Methods $12: 7-8$

Yang J et al (2020) Effectiveness, immunogenicity, and safety of influenza vaccines with MF59 adjuvant in healthy people of different age groups: a systematic review and meta-analysis. Medicine 99:e19095

Yao B, Zhang L, Liang S, Zhang C (2012) SVMTriP: a method to predict antigenic epitopes using support vector machine to integrate tri-peptide similarity and propensity. PLoS ONE 7:e45152

Zahn M, D'agostino T, Eren E, Baslé A, Ceccarelli M, Van Den Berg B (2015) Small-molecule transport by CarO, an abundant eightstranded $\beta$-barrel outer membrane protein from Acinetobacter baumannii. J Mol Biol 427:2329-2339

Zhang Y (2008) I-TASSER server for protein 3D structure prediction. BMC Bioinform 9:1-8

Publisher's Note Springer Nature remains neutral with regard to jurisdictional claims in published maps and institutional affiliations. 